\section{COMPREHENSIVE MONITORING REPORT FOR UNDERGROUND STORAGE TANKS 0439-U, 0440-U, 2073-U, 2074-U, AND 2075-U AT THE EAST END FUEL STATION, BUILDINGS 9754 AND 9754-2}

\author{
OAK RIDGE Y-12 PLANT \\ OAK RIDGE, TENNESSEE \\ FACILITY ID \#0-010117

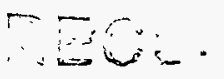 \\ June 1996 \\ DEC 201896 \\ OSTI
}

NITTINITON OF THS DOCUMENT IS UNLIMITEO 


\section{DISCLAIMER}

This report was prepared as an account of work sponsored by an agency of the United States Government. Neither the United States Government nor any agency thereof, nor any of their employees, makes any warranty, express or implied, or assumes any legal liability or responsibility for the accuracy, completeness, or usefulness of any information, apparatus, product, or process disclosed, or represents that its use would not infringe privately owned rights. Reference herein to any specific commercial product, process, or service by trade name, trademark, manufacturer, or otherwise, does not necessarily constitute or imply its endorsement, recommendation, or favoring by the United States Government or any agency thereof. The views and opinions of authors expressed herein do not necessarily state or reflect those of the United States Government or any agency thereof. 


\title{
COMPREHENSIVE MONITORING REPORT FOR UNDERGROUND STORAGE TANKS 0439-U, 0440-U, 2073-U, 2074-U, AND 2075-U AT THE EAST END FUEL STATION, BUILDINGS 9754 AND 9754-2
}

\author{
OAK RIDGE Y-12 PLANT \\ OAK RIDGE, TENNESSEE \\ FACILITY ID \#0-010117
}

\author{
June 1996 \\ Environmental Management Department \\ Health, Safety, Environment, \\ and Accountability Organization
}

\section{Prepared by}

Science Applications International Corporation Under Subcontract 22B-99069C

$$
\text { for the }
$$

Oak Ridge Y-12 Plant

Oak Ridge, Tennessee 37831

\section{Managed by}

Lockheed Martin Energy Systems, Inc.

for the

U.S. Department of Energy

Under Contract No. DE-AC05-840R21400 


\section{DISCLAIMER}

Portions of this document may be illegible in electronic image products. Images are produced from the best available original document. 


\section{TABLE OF CONTENTS}

LIST OF FIGURES

Page

LIST OF TABLES

iv

LIST OF ACRONYMS

COMPREHENSIVE MONITORING REPORT

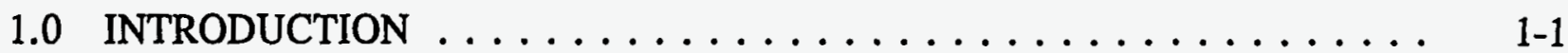

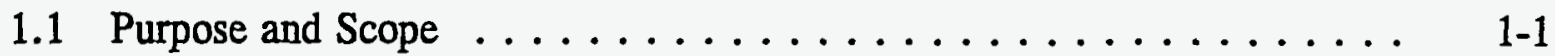

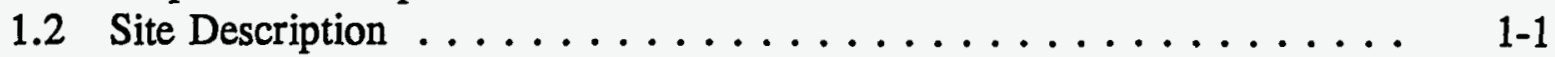

2.0 GROUNDWATER MONITORING $\ldots \ldots \ldots \ldots \ldots \ldots \ldots$ 2-1

2.1 Groundwater Measurement, Sample Collection and Analysis . . . . . . 2-1

2.1.1 Well Locations .................. 2-1

2.1.2 Groundwater Measurement and Sampling ......... 2-1

2.1.3 Sample Analysis . . . . . . . . . . . . . 2-1

2.2 Potentiometric Data . . . . . . . . . . . . . . . . 2-1

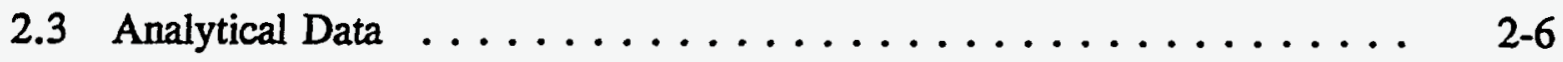

3.0 VAPOR MONITORING $\ldots \ldots \ldots \ldots \ldots \ldots \ldots \ldots \ldots \ldots \ldots$ 3-1

3.1 Monitoring Method and Locations $\ldots \ldots \ldots \ldots \ldots \ldots \ldots$ 3-1

3.2 Vapor Monitoring Results $\ldots \ldots \ldots \ldots \ldots \ldots \ldots \ldots \ldots \ldots \ldots \ldots$

REFERENCES

APPENDICES

Appendix A Laboratory Analytical Results for Comprehensive Monitoring. . . . . A-1 


\section{LIST OF FIGURES}

Figure Title $\quad$ Page

1-1 Location of the East End Fuel Station at the Y-12 Plant . . . . . . . . . 1-2

1-2 East End Fuel Station Site Map . . . . . . . . . . . . . . . . . 1-3

2-1 East End Fuel Station Site Monitoring Well Location Map . . . . . . . . 2-2

2-2 East End Fuel Station Groundwater Potentiometric $\ldots \ldots \ldots \ldots \ldots \ldots \ldots \ldots$. . . . . . . . . . . . . . . . . .

2-3 East End Fuel Station Groundwater Potentiometric $\ldots \ldots \ldots$ 2-5

2-4 Horizontal Extent of Benzene Contamination in

2-5 Horizontal Extent of TPH Contamination in

2-6 Maximum Analytical Results for TPH and BTEX in Soils at the East End Fuel Station . . . . . . . . . . . . . . . . 2-13

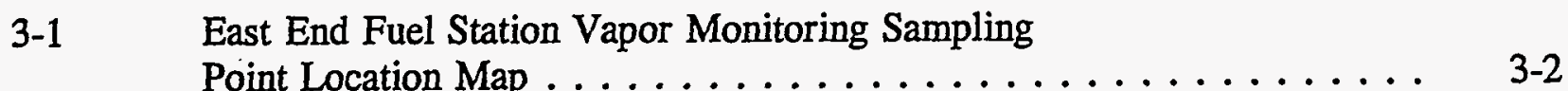

\section{TABLES}

Table Title $\quad$ Page

1-1 Tank contents and capacities at the East End Fuel Station . . . . . . . . 1-4

2-1 Water Level Measurements at the East End Fuel Station for the Periods of December 1993 and May 1996 . . . . . . . . . . 2-3

2-2 Analytical Results for Groundwater Samples Collected During Comprehensive Monitoring and Corrective Action Baseline Sampling . . . . . . . . . . . . . . . . . . 2-7 2... 2

3-1 Vapor Monitoring Results for the East End Fuel Station ........ 3-3 


\section{LIST OF ACRONYMS}

BGL

BTEX

BTOC

CAP

LEL

MSL

TDEC

TOC

TPH-DRO TPH-GRO

UST below ground level

benzene, toluene, ethylbenzene, and xylene

below top of casing

Corrective Action Plan

lower explosive limit

mean sea level

Tennessee Department of Environment and Conservation

top of casing

total petroleum hydrocarbons - diesel range organics

total petroleum hydrocarbons - gasoline range organics

underground storage tank 


\section{SIGNATURE PAGE FOR THE \\ COMPREHENSIVE MONITORING REPORT \\ FOR UNDERGROUND STORAGE TANKS 0439-U, 0440-U, 2073-U, 2074-7, AND \\ 2068-U AT THE EAST END FUEL STATION, BUILDINGS 9754 AND 9754-2}

I certify under penalty of law, including but not limited to penalties for perjury, that the information contained in this report and on any attachments, is true, accurate, and complete to the best of my knowledge, information, and belief. I am aware that there are significant penalties for submitting false information, including the possibility of fine and imprisonment for intentional violations.

See Attached Certification

Owner/Operator (Print)

William D. Keefer

P.E. or P.G. (Print)

If a P.E. signs this report, please indicate the area of expertise.

(Print or Type)

$\overline{\text { Signature }} \overline{\text { Date }}$
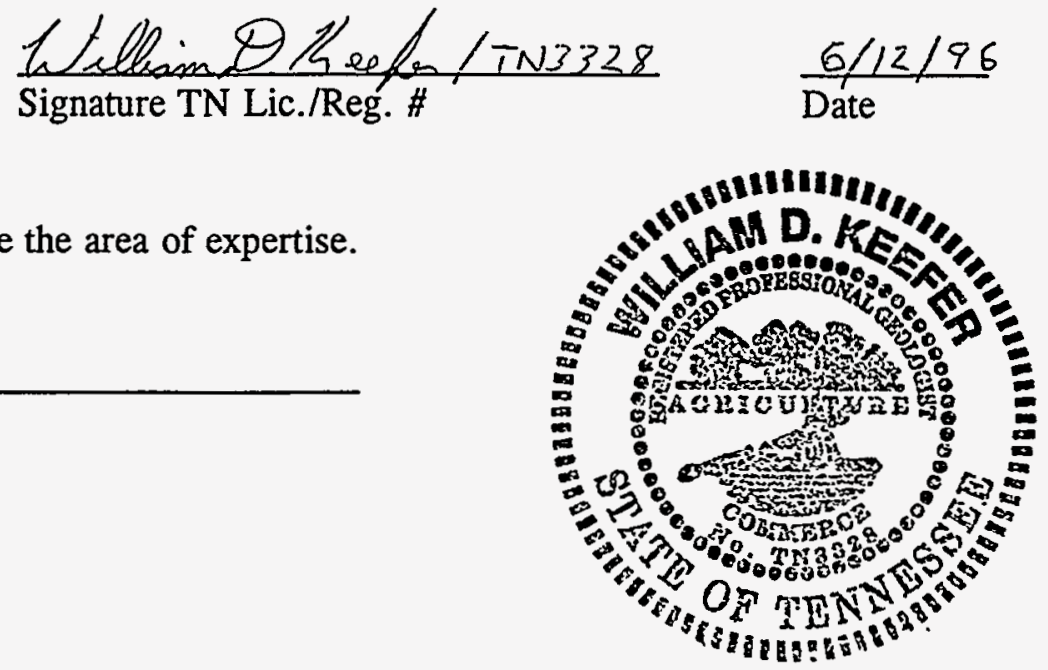

(P.E./P.G. Stamp/Seal)

County of Anderence State of Lennisece

Subscribed and Sworn to before me this

12 day of

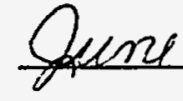
1996

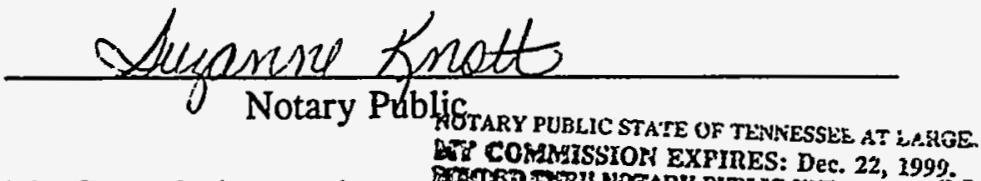

My Commission expires: 


\subsection{INTRODUCTION}

\subsection{PURPOSE AND SCOPE}

The purpose of this document is to provide hydrogeologic, geochemical, and vapor monitoring data required for comprehensive monitoring of underground storage tanks (UST) 0439-U, 0440-U, 2073-U, 2074-U, and 2075-U at the East End Fuel Station. Comprehensive monitoring was conducted at the site in May 1996 at the direction of the Tennessee Department of Environment and Conservation (TDEC) based on review and notice of deficiencies in the Site-specific Standard Request (letter from Jeffrey Fields to Robert Spence, dated May 6, 1996). Comprehensive monitoring and preparation of this report have been conducted in accordance with the requirements of the TDEC Rule 1200-1-15 and the TDEC UST Reference Handbook, Second Edition (TDEC 1994).

This document is organized into three sections. Section 1 presents introductory information relative to the site including regulatory initiative and a description of the site. Section 2 includes the results of water level measurement and sampling of monitoring wells at the site. Section 3 presents data from vapor monitoring conducted in subsurface utilities present at the site. Appendix A contains the original analytical laboratory results for environmental and quality control samples collected during comprehensive monitoring.

\subsection{SITE DESCRIPTION}

The East End Fuel Station is located at the eastern edge of the Oak Ridge Y-12 Plant in Oak Ridge, Tennessee (Figure 1-1). The site occurs in a moderately industrialized area on the north side of First Street between "A" Road and Agate Road (Figure 1-2). The site includes Building 9754-2 and the former Building 9754. Tanks 0439-U and 0440-U were located adjacent to Building 9754-2, and tanks 2073-U, 2074-U, and 2075-U serviced pump islands adjacent to the former Building 9754. The tank contents and capacities are summarized in Table 1-1. This area is characterized by a gentle slope stepping down to the south toward Upper East Fork Poplar Creek.

The East End Fuel Station has been in use as a primary fueling point for the Y-12 Plant since 1944. The holding tanks at former Building 9754 facility were in use until 1978 when they were emptied and taken out of service. At that time, the piping associated with the holding tanks and two emergency dispensing pumps were capped at a valving station and abandoned in place. In 1993, tanks 2073-U, 2074-U, 2075-U and associated piping were excavated and removed. The USTs at the Building 9754-2 facility were put into service in 1979 and taken out of service and removed in June 1989. The East End Fuel Station is still in use and is currently supplied by two aboveground storage tanks. 


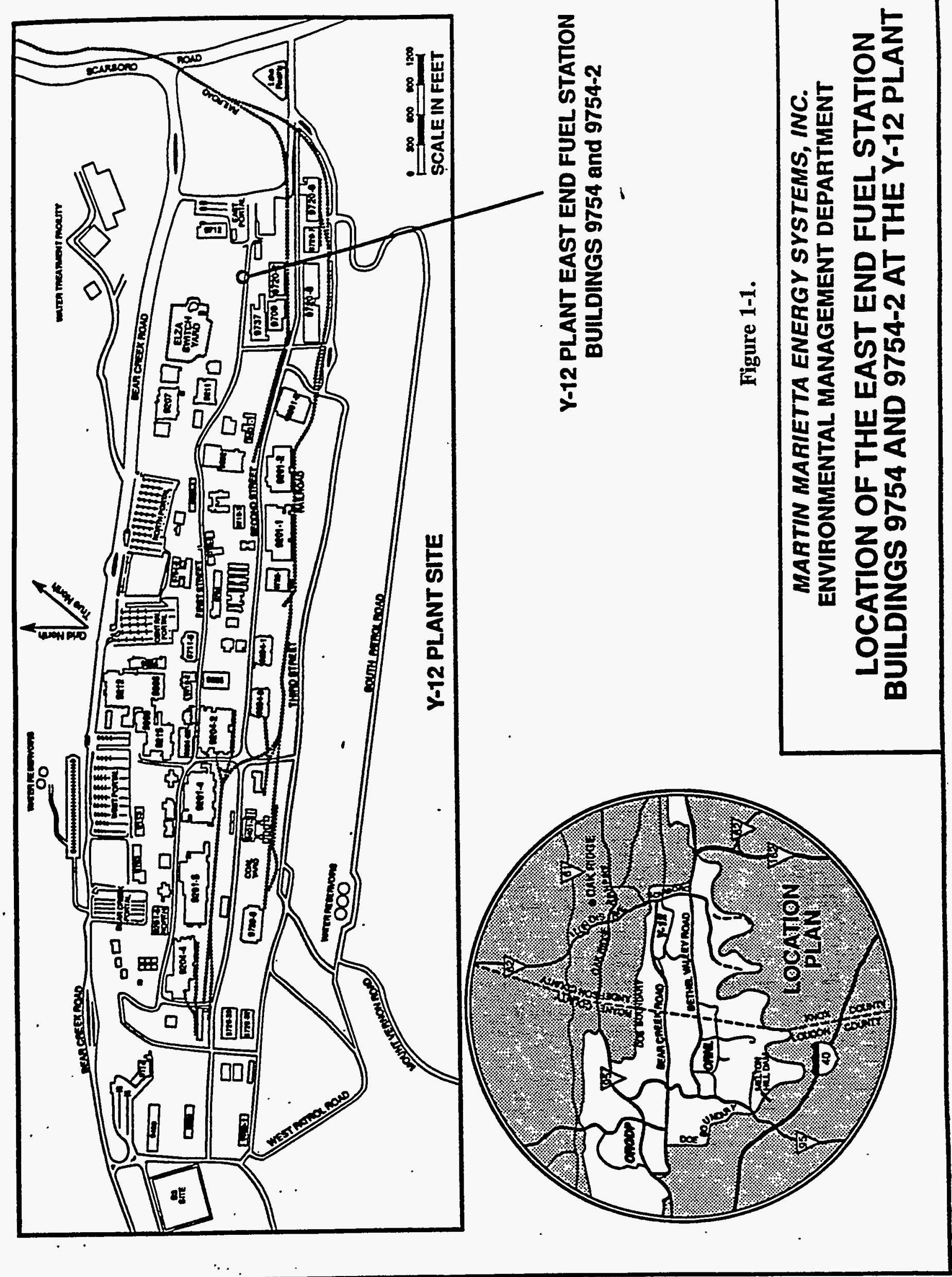




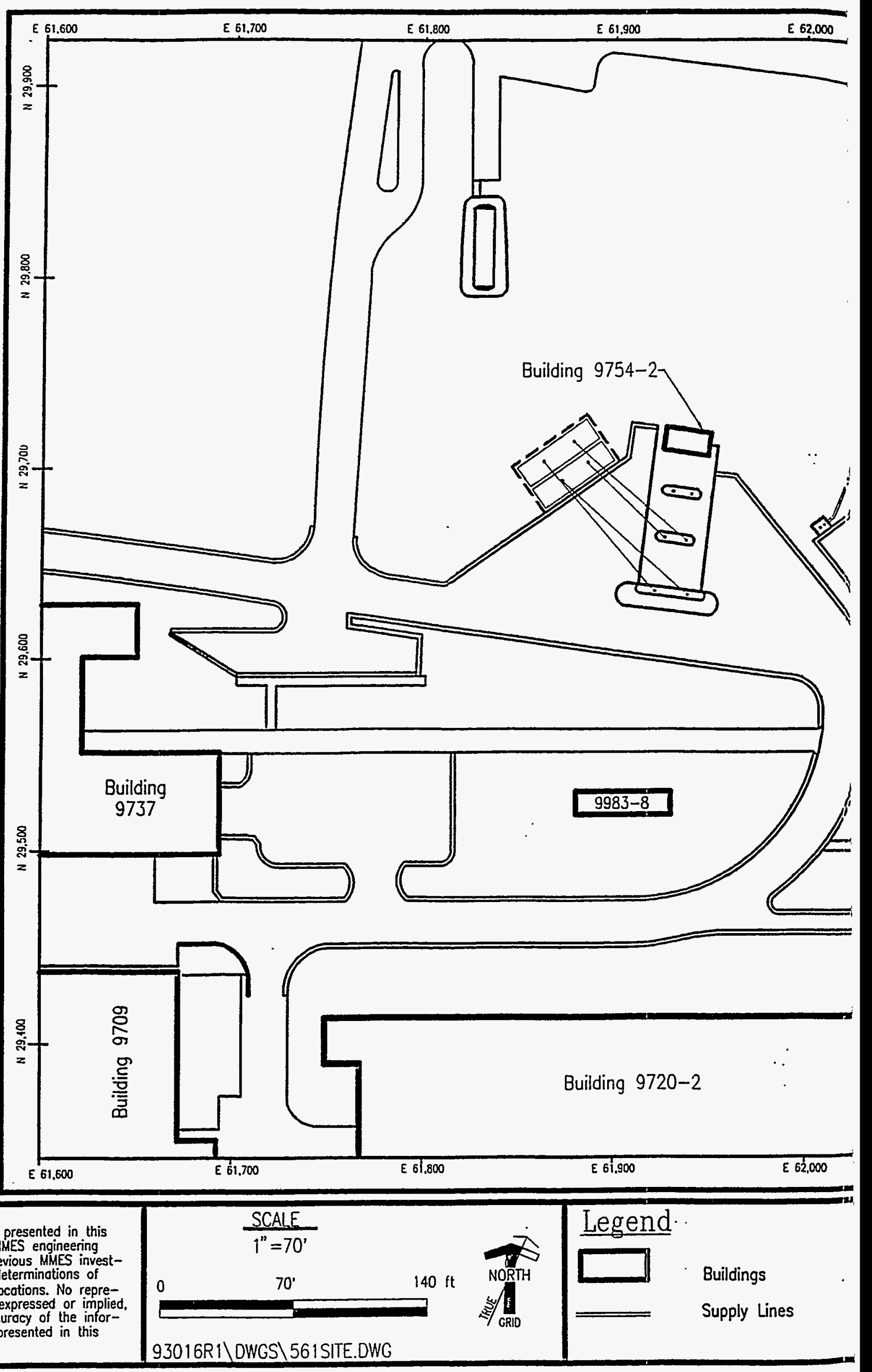

All location informotion presented in this figure is bosed upon MMES engineering drowings, results of previous MMES investigations, and/or field determinations of feature and sampling locations. No representation or worranty, expressed or implied, is made os to the accuracy of the information or stolements presented in this figure. 
Table 1-1. Tank contents and capacities at the East End Fuel Station

\begin{tabular}{|c|c|c|}
\hline Tank & $\begin{array}{l}\text { Capacity } \\
\text { (gallons) }\end{array}$ & Contents \\
\hline $0439-U$ & 20,000 & Gasoline \\
\hline $0440-U$ & 10,000 & Diesel \\
\hline $2073-U$ & 1,000 & Gasoline \\
\hline 2074-U & 1,000 & Gasoline \\
\hline $2068-U$ & 1,000 & Diesel \\
\hline
\end{tabular}




\subsection{GROUNDWATER MONITORING}

\subsection{GROUNDWATER MEASUREMENT, SAMPLE COLLECTION, AND ANALYSIS}

\subsubsection{Well Locations}

Twelve monitoring wells at the site, including GW-183, GW-281, GW-282, GW-283, GW-284, GW-285, GW-658, GW-659, GW-767, GW-802, GW-803, and GW-804 were sampled during comprehensive monitoring. The locations of these wells are presented in Figure 2-1. Well installation reports and construction details for these wells are presented in the Corrective Action Plan (CAP) for the Site (Corrective Action Plan for Underground Storage Tanks 0439-U, 0440-U, 2073-U, 2074-U, and 2075-U at the East End Fuel Station, Buildings 9754 and 9754-2, Appendix E, Lockheed Martin Energy Systems, Inc., Y/SUB/92-99928C/2) (Energy Systems 1992) and in the Corrective Action Baseline Report (Corrective Action Baseline Report for Underground Storage Tanks 0439-U, 0440-U, 2073-U, 2074-U, and 2075-U at the East End Fuel Station, Buildings 9754 and 9754-2, Appendix A, Lockheed Martin Energy Systems, Inc., Y/SUB/94-99069C/Y15/1) (Energy Systems 1994).

\subsubsection{Groundwater Measurement and Sampling}

Groundwater level measurement and analytical sampling were conducted for the specified monitoring wells at the East End Fuel Station on May 12, 1996. Prior to sampling, water levels were measured to the nearest $0.01 \mathrm{ft}$ using an electronic water level indicator. The wells were then purged of approximately three well volumes. Field measurements of $\mathrm{pH}$, conductivity, temperature, and dissolved oxygen were conducted during purging to ensure representativeness for sampling. The wells were allowed to recover and analytical samples were collected using a bailer. Samples were placed into pre-cleaned containers with no headspace. During water level measurement and purging, an oily sheen was noted as visible in GW-658 and as slightly visible in GW-183. Purge water from well GW-658 was noted as muddy/sandy and recovery in monitoring wells GW-281 and GW-282 was slow.

\subsubsection{Sample Analysis}

Samples were analyzed for total petroleum hydrocarbons (TPH) gasoline range organics (TPH-GRO), diesel range organics (TPH-DRO), and for benzene, toluene, ethylbenzene, and xylene (BTEX).

\subsection{POTENTIOMETRIC DATA}

Potentiometric data collected during baseline sampling (conducted December 18, 1993) and comprehensive monitoring are presented in Table 2-1. Figures 2-2 and 2-3 present potentiometric maps from these two periods of sampling. These figures illustrate that 
Table 2-1. Water Level Measurements at the East End Fuel Station for the Periods of December 1993 and May 1996

\begin{tabular}{|c|c|c|c|c|c|}
\hline $\begin{array}{c}\text { Monitoring } \\
\text { Well \# }\end{array}$ & $\begin{array}{c}\text { Date } \\
\text { Measured }\end{array}$ & $\begin{array}{c}\text { Total Well } \\
\text { Depth (BGL) }\end{array}$ & $\begin{array}{l}\text { Top of } \\
\text { Casing } \\
(\mathrm{MSL})^{2}\end{array}$ & $\begin{array}{c}\text { Top of } \\
\text { Casing to } \\
\text { Water Level } \\
\text { (BTOC) }^{3}\end{array}$ & $\begin{array}{l}\text { Potentiometric } \\
\text { Surface } \\
\text { (MSL) }\end{array}$ \\
\hline GW-183 & $\begin{array}{l}12 / 18 / 93 \\
05 / 12 / 96\end{array}$ & $\begin{array}{l}30.6 \mathrm{ft} \\
30.6 \mathrm{ft}\end{array}$ & $\begin{array}{l}946.02 \mathrm{ft} \\
946.02 \mathrm{ft}\end{array}$ & $\begin{array}{r}9.9 \mathrm{ft} \\
10.5 \mathrm{ft}\end{array}$ & $\begin{array}{l}936.12 \mathrm{ft} \\
935.52 \mathrm{ft}\end{array}$ \\
\hline GW-281 & $\begin{array}{l}12 / 18 / 93 \\
05 / 12 / 96\end{array}$ & $\begin{array}{l}17.5 \mathrm{ft} \\
17.5 \mathrm{ft}\end{array}$ & $\begin{array}{l}.946 .08 \mathrm{ft} \\
946.08 \mathrm{ft}\end{array}$ & $\begin{array}{l}5.7 \mathrm{ft} \\
5.8 \mathrm{ft}\end{array}$ & $\begin{array}{l}940.38 \mathrm{ft} \\
940.28 \mathrm{ft}\end{array}$ \\
\hline GW-282 & $\begin{array}{l}12 / 18 / 93 \\
05 / 12 / 96\end{array}$ & $\begin{array}{l}16.0 \mathrm{ft} \\
16.0 \mathrm{ft}\end{array}$ & $\begin{array}{l}943.94 \mathrm{ft} \\
943.94 \mathrm{ft}\end{array}$ & $\begin{array}{r}1.9 \mathrm{ft} \\
8.63 \mathrm{ft}\end{array}$ & $\begin{array}{l}942.04 \mathrm{ft} \\
935.31 \mathrm{ft}\end{array}$ \\
\hline GW-283 & $\begin{array}{l}12 / 18 / 93 \\
05 / 12 / 96\end{array}$ & $\begin{array}{l}20.0 \mathrm{ft} \\
20.0 \mathrm{ft}\end{array}$ & $\begin{array}{l}938.62 \mathrm{ft} \\
938.62 \mathrm{ft}\end{array}$ & $\begin{array}{l}5.4 \mathrm{ft} \\
5.8 \mathrm{ft}\end{array}$ & $\begin{array}{l}933.22 \mathrm{ft} \\
932.82 \mathrm{ft}\end{array}$ \\
\hline GW-284 & $\begin{array}{l}12 / 18 / 93 \\
05 / 12 / 96\end{array}$ & $\begin{array}{l}18.0 \mathrm{ft} \\
18.0 \mathrm{ft}\end{array}$ & $\begin{array}{l}941.61 \mathrm{ft} \\
941.61 \mathrm{ft}\end{array}$ & $\begin{array}{r}8.2 \mathrm{ft} \\
8.85 \mathrm{ft}\end{array}$ & $\begin{array}{l}933.41 \mathrm{ft} \\
932.76 \mathrm{ft}\end{array}$ \\
\hline GW-285 & $\begin{array}{l}12 / 18 / 93 \\
05 / 12 / 96\end{array}$ & $\begin{array}{l}21.0 \mathrm{ft} \\
21.0 \mathrm{ft}\end{array}$ & $\begin{array}{l}942.46 \mathrm{ft} \\
942.46 \mathrm{ft}\end{array}$ & $\begin{array}{l}9.1 \mathrm{ft} \\
9.5 \mathrm{ft}\end{array}$ & $\begin{array}{l}933.36 \mathrm{ft} \\
932.96 \mathrm{ft}\end{array}$ \\
\hline GW-658 & $\begin{array}{l}12 / 18 / 93 \\
05 / 12 / 96\end{array}$ & $\begin{array}{l}19.1 \mathrm{ft} \\
19.1 \mathrm{ft}\end{array}$ & $\begin{array}{l}944.81 \mathrm{ft} \\
944.81 \mathrm{ft}\end{array}$ & $\begin{array}{r}10.6 \mathrm{ft} \\
11.65 \mathrm{ft}\end{array}$ & $\begin{array}{l}934.21 \mathrm{ft} \\
933.16 \mathrm{ft}\end{array}$ \\
\hline GW-659 & $\begin{array}{l}12 / 18 / 93 \\
05 / 12 / 96\end{array}$ & $\begin{array}{l}16.7 \mathrm{ft} \\
16.7 \mathrm{ft}\end{array}$ & $\begin{array}{l}941.16 \mathrm{ft} \\
941.16 \mathrm{ft}\end{array}$ & $\begin{array}{r}6.9 \mathrm{ft} \\
7.75 \mathrm{ft}\end{array}$ & $\begin{array}{l}934.26 \mathrm{ft} \\
933.41 \mathrm{ft}\end{array}$ \\
\hline GW-767 & $\begin{array}{l}12 / 18 / 93 \\
05 / 12 / 96\end{array}$ & $\begin{array}{l}18.0 \mathrm{ft} \\
18.0 \mathrm{ft}\end{array}$ & $\begin{array}{l}948.54 \mathrm{ft} \\
948.54 \mathrm{ft}\end{array}$ & $\begin{array}{l}10.9 \mathrm{ft} \\
12.0 \mathrm{ft}\end{array}$ & $\begin{array}{l}937.64 \mathrm{ft} \\
936.54 \mathrm{ft}\end{array}$ \\
\hline GW-802 & $\begin{array}{l}12 / 18 / 93 \\
05 / 12 / 96\end{array}$ & $\begin{array}{l}26.5 \mathrm{ft} \\
26.5 \mathrm{ft}\end{array}$ & $\begin{array}{l}941.83 \mathrm{ft} \\
941.83 \mathrm{ft}\end{array}$ & $\begin{array}{l}4.9 \mathrm{ft} \\
6.5 \mathrm{ft}\end{array}$ & $\begin{array}{l}936.93 \mathrm{ft} \\
935.33 \mathrm{ft}\end{array}$ \\
\hline GW-803 & $\begin{array}{l}12 / 18 / 93 \\
05 / 12 / 96\end{array}$ & $\begin{array}{l}25.0 \mathrm{ft} \\
25.0 \mathrm{ft}\end{array}$ & $\begin{array}{l}942.53 \mathrm{ft} \\
942.53 \mathrm{ft}\end{array}$ & $\begin{array}{l}9.2 \mathrm{ft} \\
9.5 \mathrm{ft}\end{array}$ & $\begin{array}{l}933.33 \mathrm{ft} \\
933.03 \mathrm{ft}\end{array}$ \\
\hline GW-804 & $\begin{array}{l}12 / 18 / 93 \\
05 / 12 / 96\end{array}$ & $\begin{array}{l}25.0 \mathrm{ft} \\
25.0 \mathrm{ft}\end{array}$ & $\begin{array}{l}942.55 \mathrm{ft} \\
942.55 \mathrm{ft}\end{array}$ & $\begin{array}{r}9.2 \mathrm{ft} \\
10.0 \mathrm{ft}\end{array}$ & $\begin{array}{l}933.35 \mathrm{ft} \\
832.55 \mathrm{ft}\end{array}$ \\
\hline
\end{tabular}




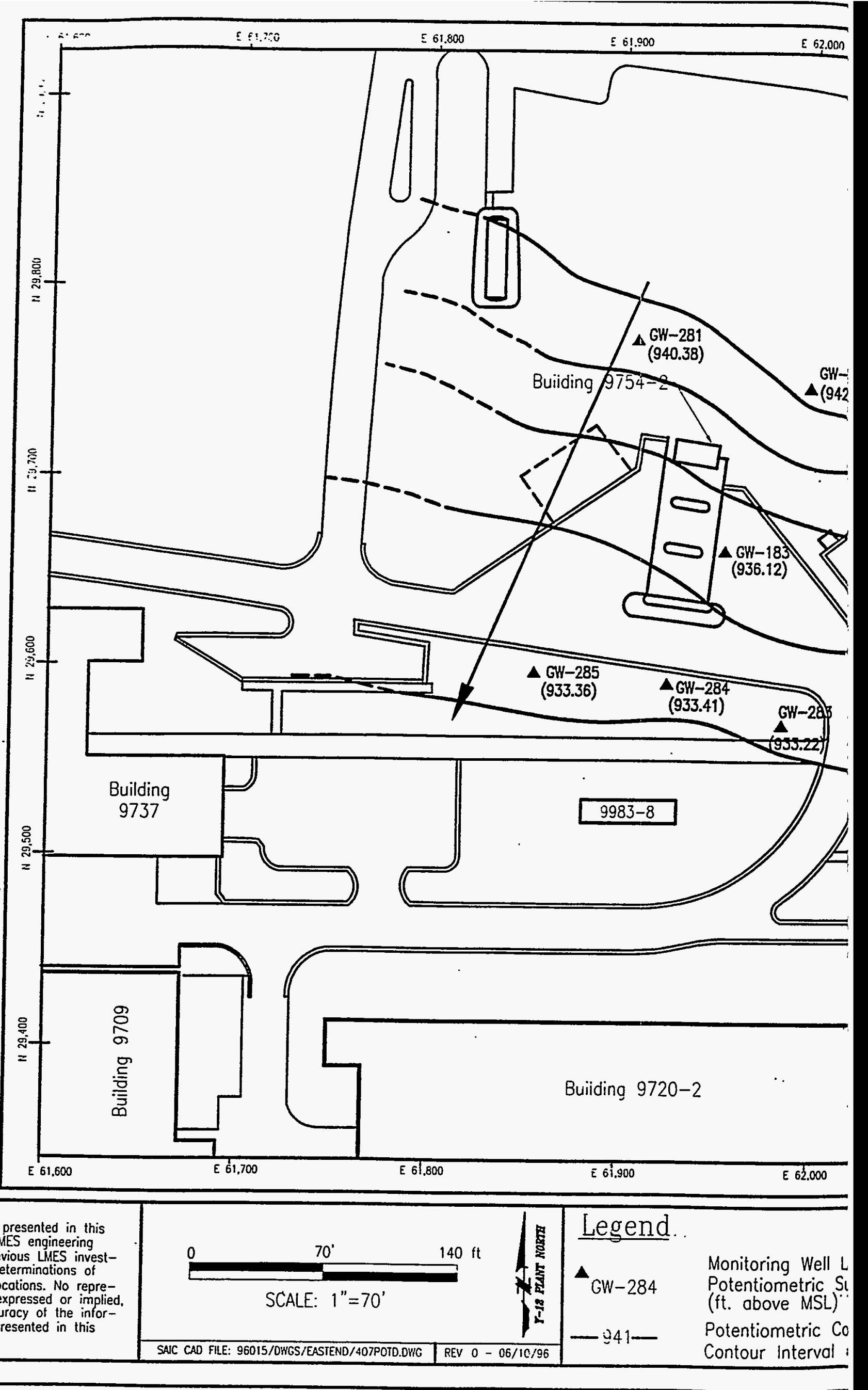


groundwater movement is generally from the north to south across the site with little seasonal variability.

\subsection{ANALYTICAL DATA}

Analytical data for groundwater sampled at the site during the 1992 and 1993 Y-12 Plant Groundwater Quality Assessment Sampling (Energy Systems 1993 and 1994a), corrective action baseline sampling (August 1993), and comprehensive monitoring (May 1996), are summarized in Table 2-2. Because monitoring wells GW-802, GW-803, and GW-804 were installed as part of the corrective action baseline investigation, only two rounds of analytical data are available for these wells. Laboratory analytical sheets for the samples collected during comprehensive monitoring are presented in Appendix A. Except where otherwise noted, sample values in Table 2-2 for total TPH represent summations of TPH-GRO and TPH-DRO values. Where a TPH-GRO or TPH-DRO result is qualified as a non-detect (U qualifier), and the detection limit is given, the detection limit is used in the summation.

The delineation of the horizontal extent of benzene contamination in groundwater, based on the results of comprehensive monitoring, is presented in Figure 2-4. The horizontal extent of TPH contamination is presented in Figure 2-5. Maximum analytical results for TPH and BTEX in soil, determined during several investigations at the site, are illustrated on Figure 2-6. Analytical data for soil are summarized in the Site-specific Standard Request for Underground Storage Tanks 0439-U, 0440-U, 2073-U, 2074U, and 2075-U at the East End Fuel Station, Buildings 9754 and 9754-2, Table B-1, Lockheed Martin Energy Systems, Inc., (Energy Systems 1994b).

Table 2-2 and Figures 2-4 and 2-5 demonstrate that petroleum contamination remains present in monitoring wells GW-183 and GW-658 above TDEC non-drinking water Closure Action Limits $0.07 \mathrm{ppm}$ for benzene and $1.0 \mathrm{ppm}$ for TPH. Both of these wells have historically produced values in excess of these closure limits. Comprehensive monitoring analytical results from GW-659 illustrate that contamination, at a level exceeding these closure limits, has not migrated downgradient to the location of this well. 
Table 2-2. Analytical Results for Groundwater Samples Collected During Comprehensive Monitoring and Corrective Action Baseline Sampling and Y-12 Plant Groundwater Quality Assessments

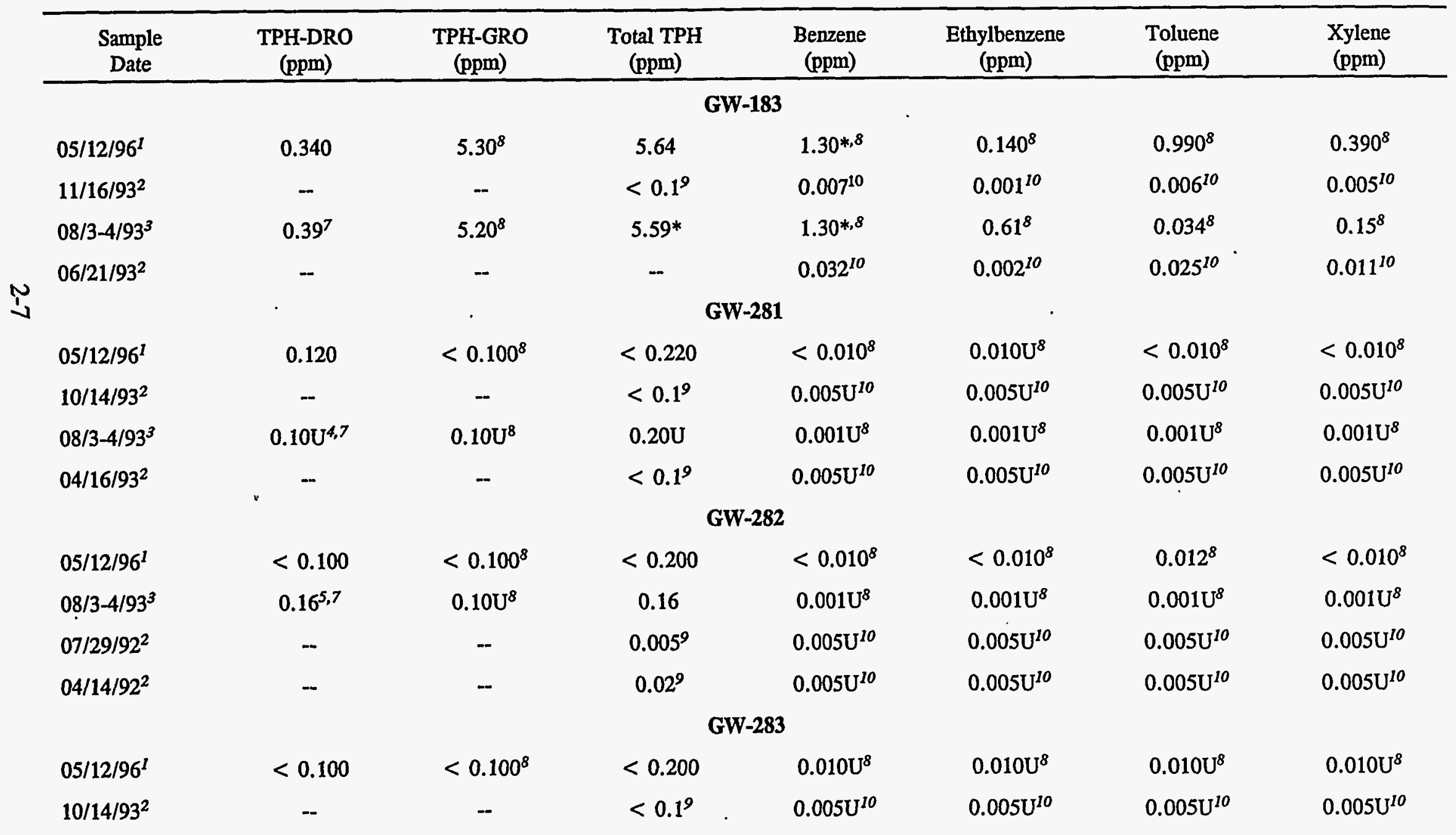




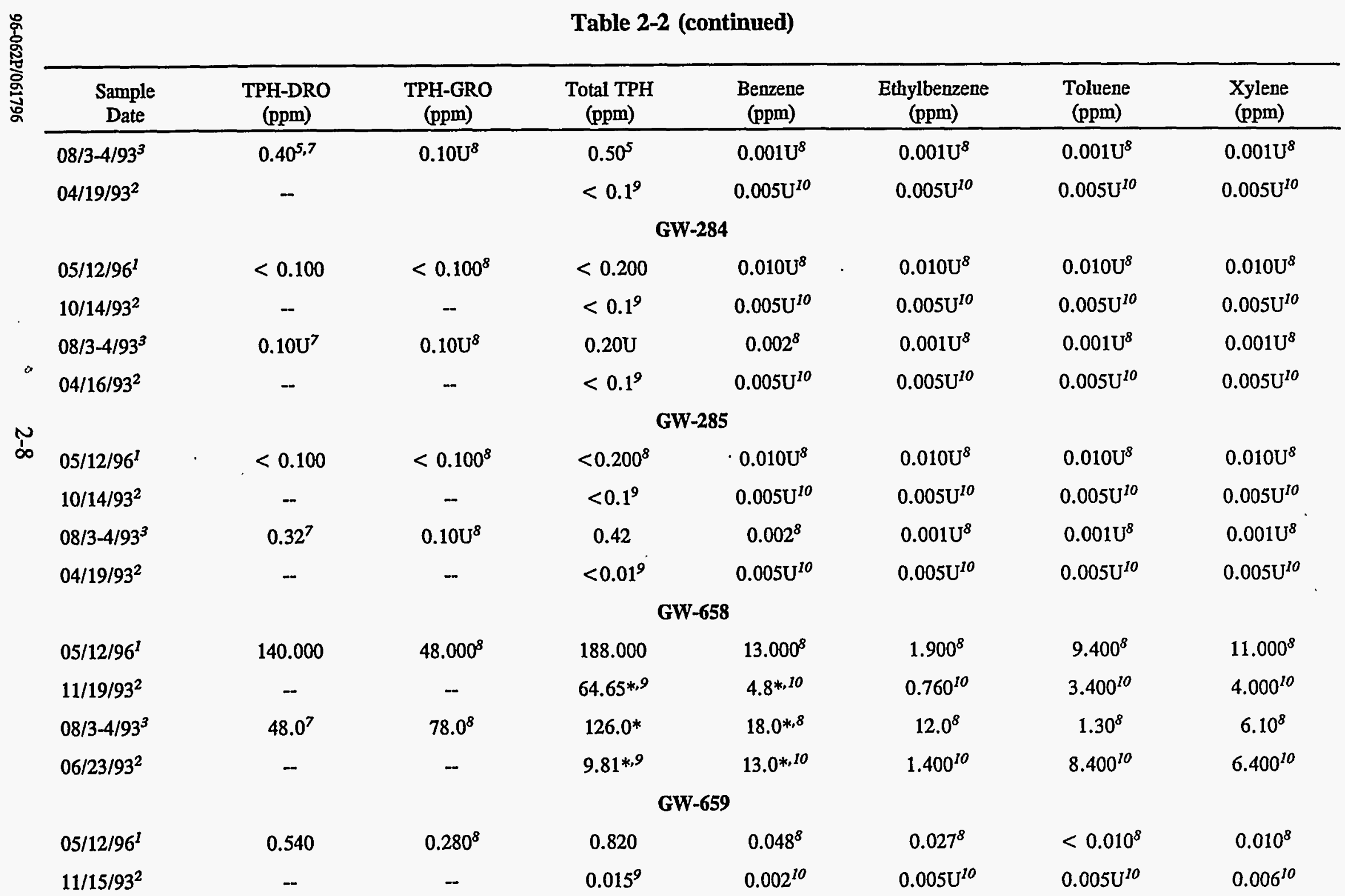




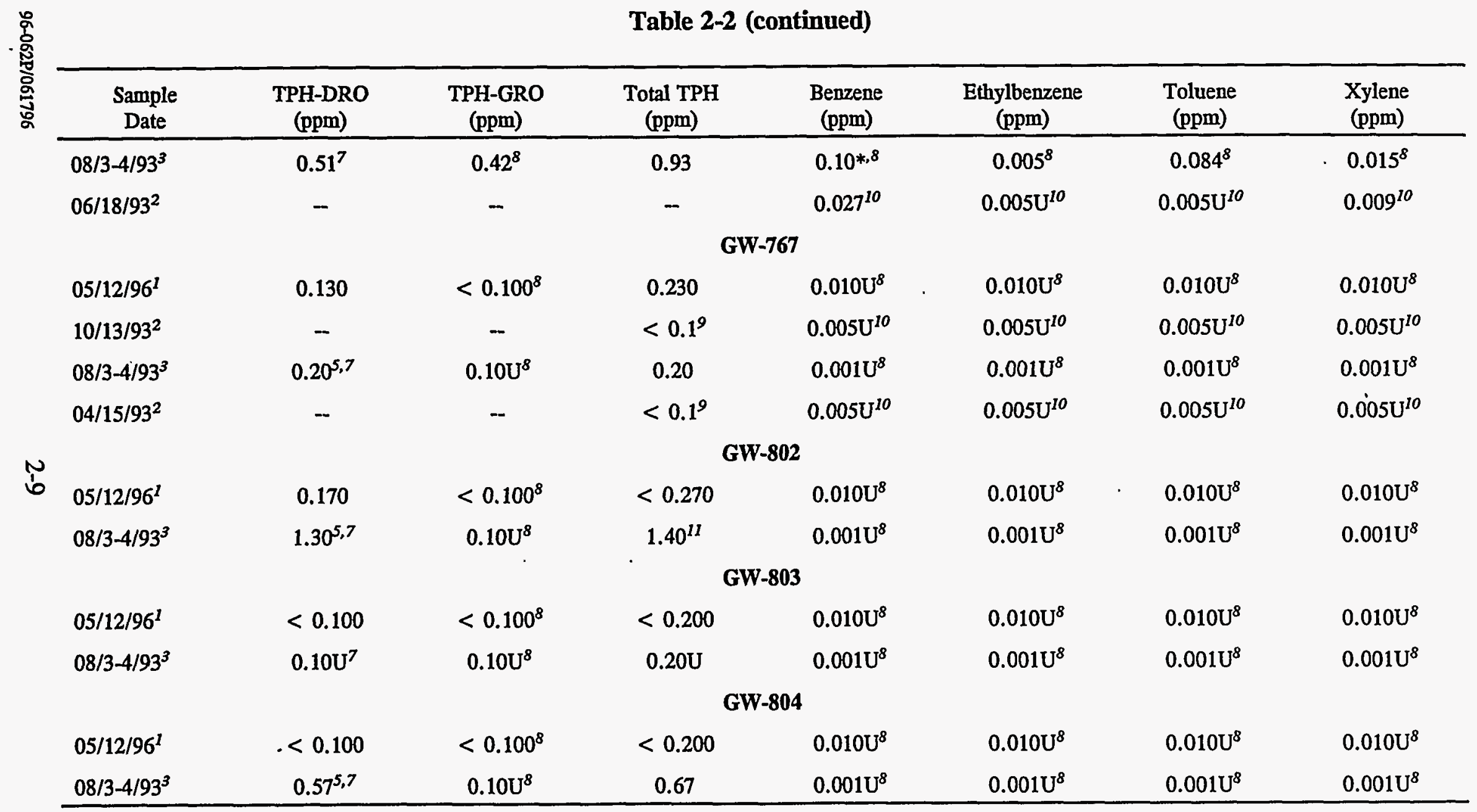

NOTES:

1 Sample collected during Comprehensive Monitoring.

2 Sample collected as part of Y-12 Plant Groundwater Quality Assessment.

3 Sample collected during Corrective Action Baseline Investigation.

4 U - Contaminant not detected above quantitation limit during analysis.

5 Analytical notation indicating a significant fraction of the detected

compounds did not have a hydrocarbon pattern.

6 ND - Contaminant not detected. 
7 Analyzed by SW-846/8015: Gas Chromatography for Nonhalogenated Volatile Organic Compounds (Modified for TPH/DRO).

8 Analyzed by SW-846/8020: Gas Chromatography for Aromatic Volatile Organic Compounds (Modified for TPH-GRO).

- Methods 8015-8020, Gas Chromatography, Modified California Method (EPA November 1986).

10 Analyzed by Gas Chromatography/Mass Spectrometry, Modified Contract Laboratory Program Protocol (EPA, February 1988).

* Indicates contaminant level greater than applicable TDEC closure action level for the East End Fuel Station, Benzene $0.070 \mathrm{ppm}$, TPH $1.0 \mathrm{ppm}$. 


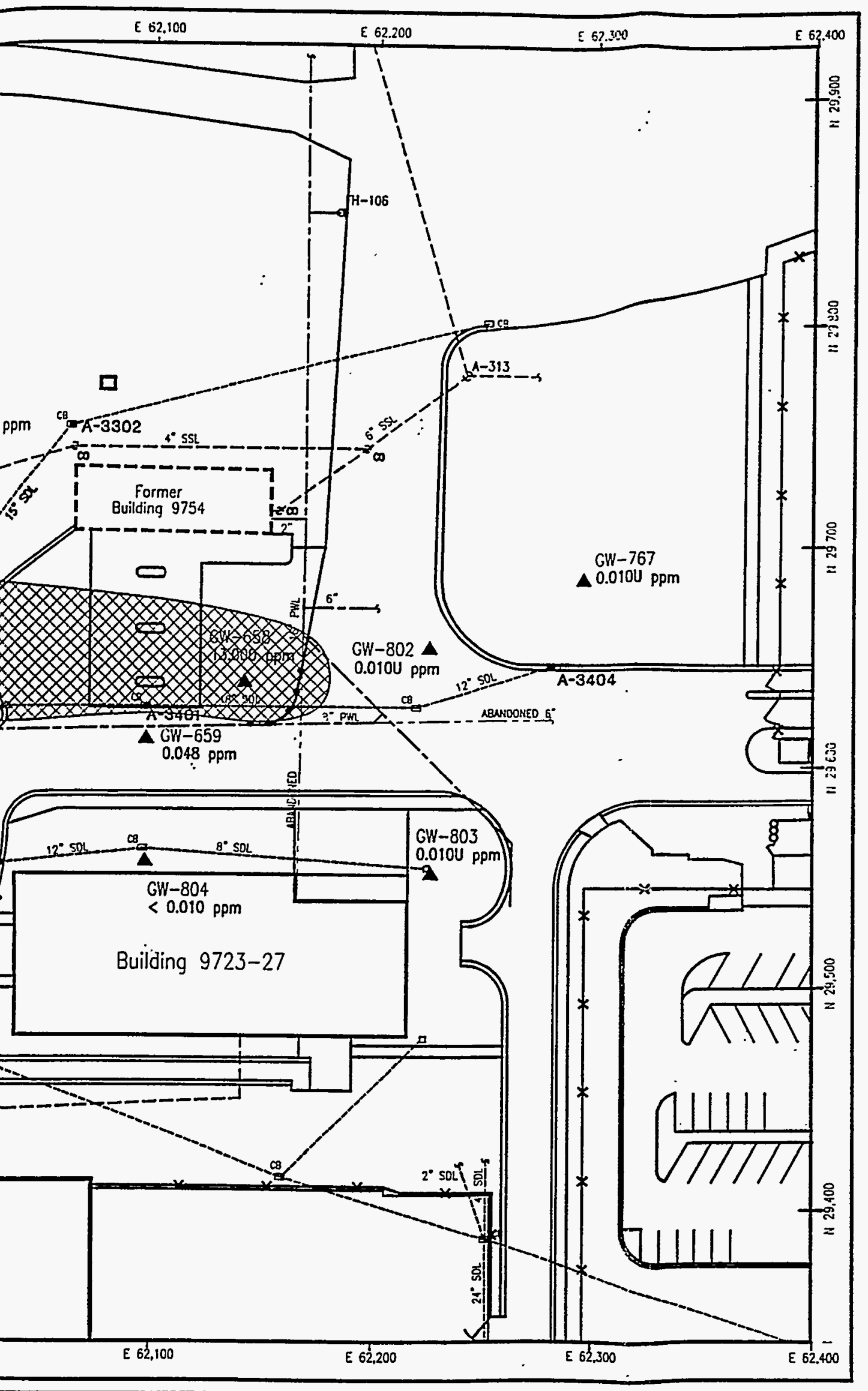

Notes:

1.) Soil contomination dota illustrated on Figure 2-6.

2.) $U$ indicales onalyte not detected obove quontitation limit.

3.) During sampling free product noted os visible in GW-658 and as slightly visible in $\mathrm{GH}-183$.

\section{SOL Underground Stormwater Orainoge Line \\ - SSL_- Sanitory Underground Sewer Line \\ _Py___ Underground Potable Water Line \\ - A-3401 Air Monitoring Locotion}

Lockheed Mortin Energy Systems, Inc. Environmental Management Department

FIGURE 2-4

Horizontal Extent of Benzene Contamination in Groundwater at the East Find Fuel Station, May 1996 


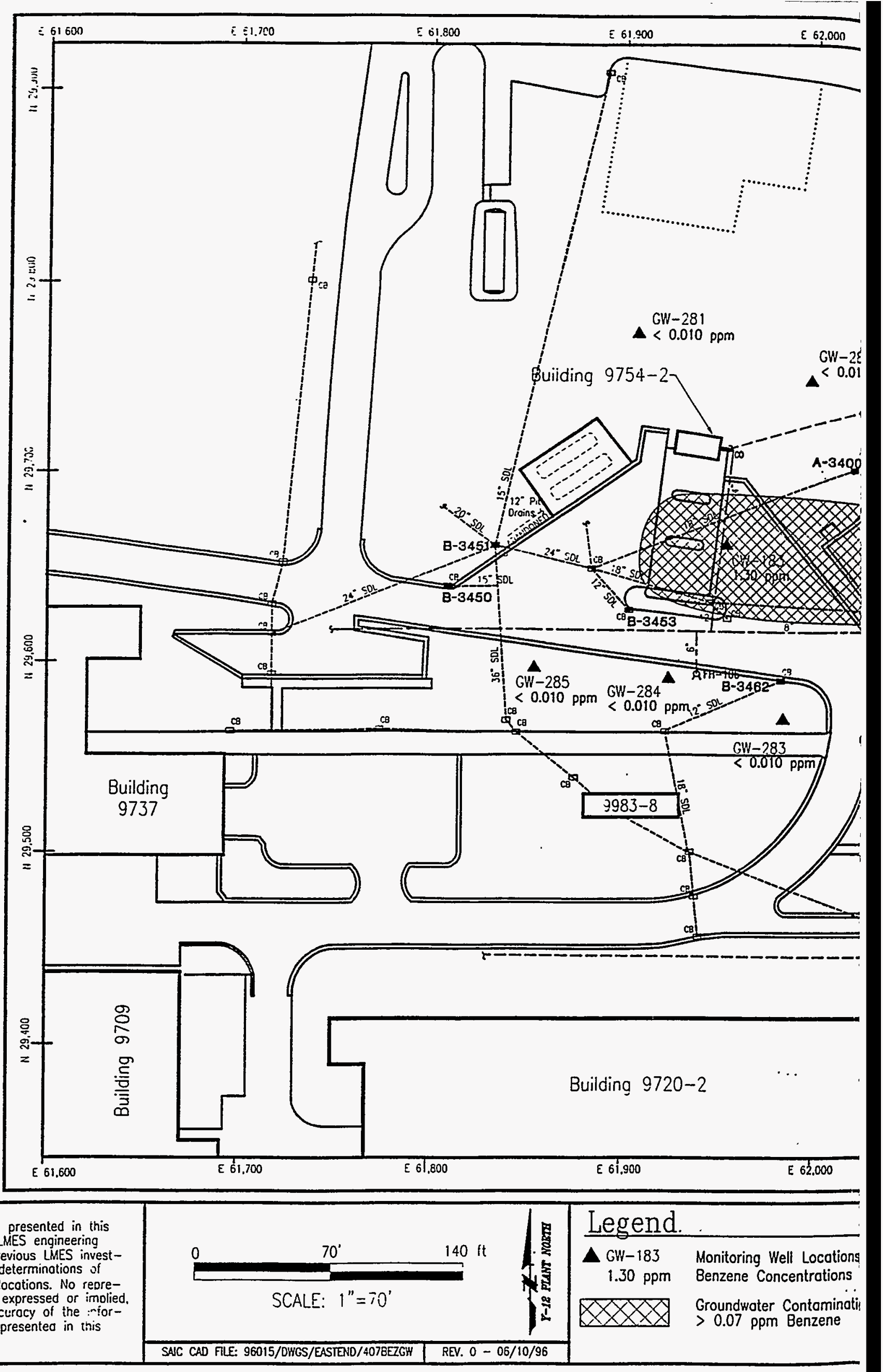




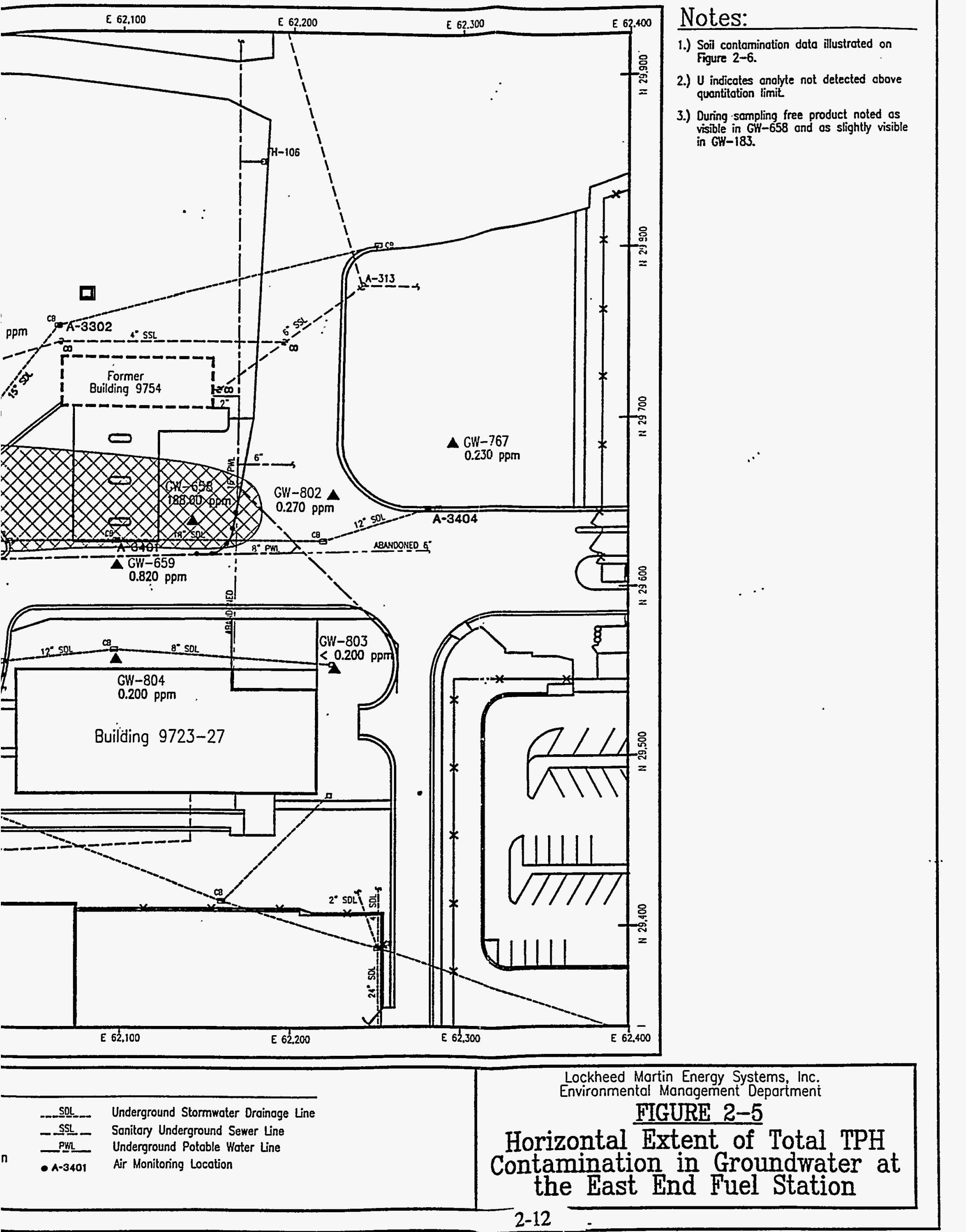




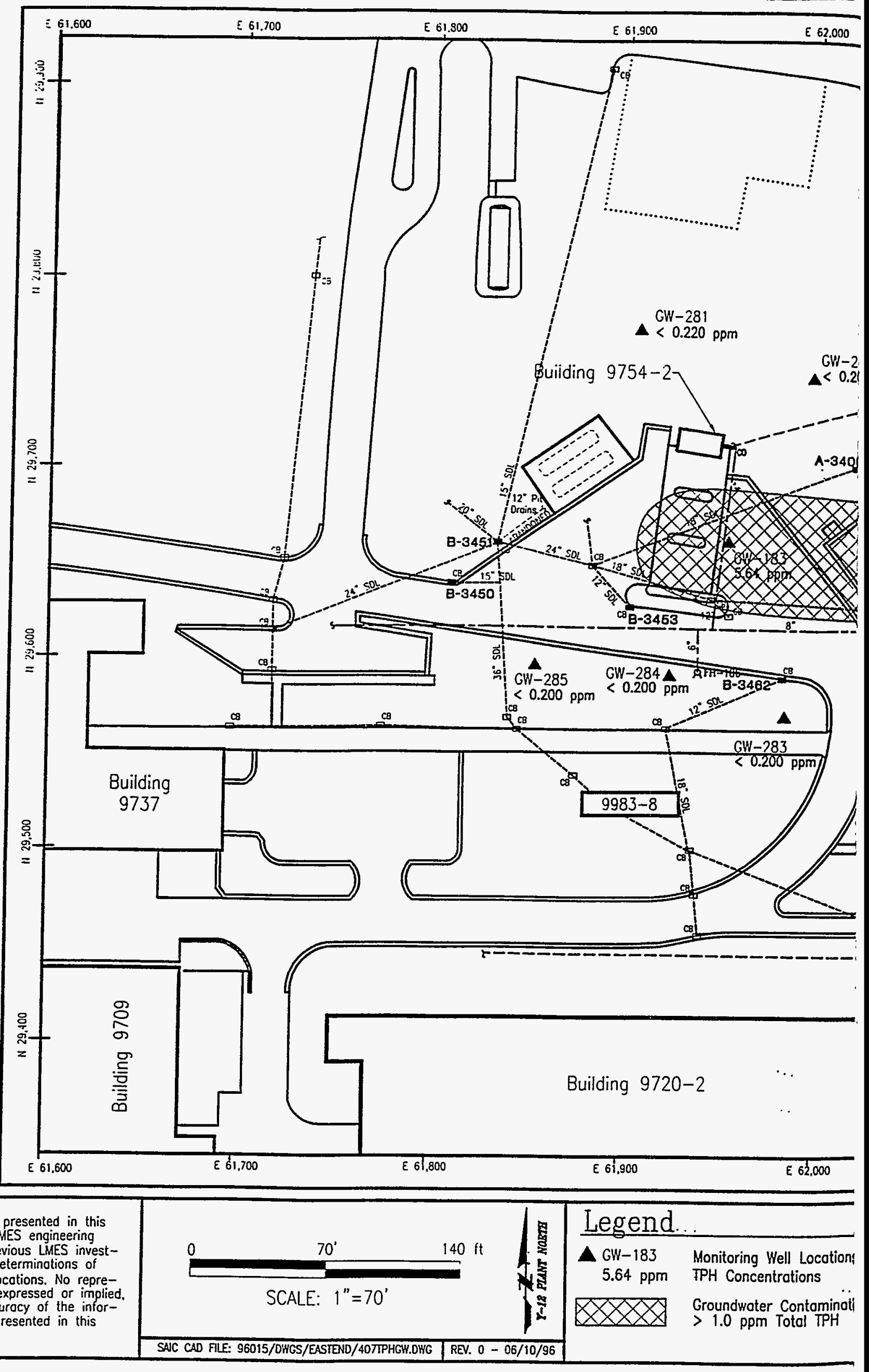




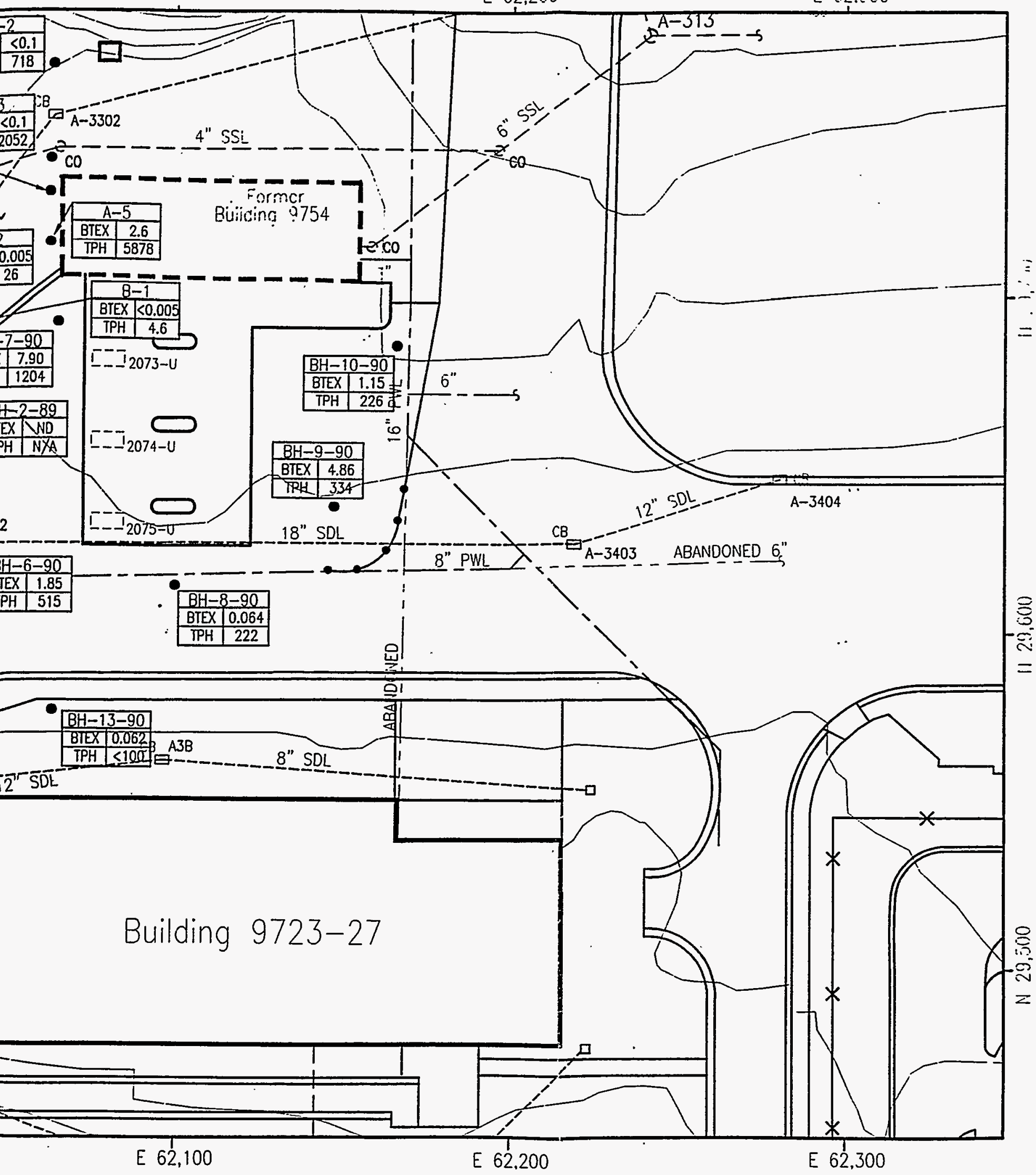

Lockheed Martin Energy Systems, Inc. Environrmental Managernenl Department

$$
\text { Figure 2-6 }
$$

- - Sanitary Underground Sewer Line

_ _ _ Underground Potable Water Line

Maximum Analytical Results for TPH \& BTEX in Soils at the East End Fuel Station 


\subsection{VAPOR MONITORING}

\subsection{MONITORING METHOD AND LOCATIONS}

Vapor monitoring as part of comprehensive monitoring was conducted on May 14, 1996. Eight access points along an interconnected stormwater drainage line network that runs across the site were monitored. The locations of sampling points are illustrated on Figure 3-1. Locations A3401 are the only accessible substructures within the delineated groundwater contamination plume.

At each location, the internal atmosphere was monitored using direct-reading instruments. Organic vapors were monitored using a Thermo Environmental ${ }^{\text {Th }}$ organic vapor meter (OVM) (serial number M249385). Oxygen and lower explosive limit (LEL) were monitored using an MSA-260 combination meter (serial number M205333).

\subsection{VAPOR MONITORING RESULTS}

The results of vapor monitoring at the East End Fuel Station are presented in Table 3-1. Measured oxygen percentages are within the range of normal air concentrations. LEL readings of $0 \%$ at all the monitored sites indicate that an explosion hazard does not exist within the monitored utilities. Organic vapor readings are all equal to $0.0 \mathrm{ppm}$. 


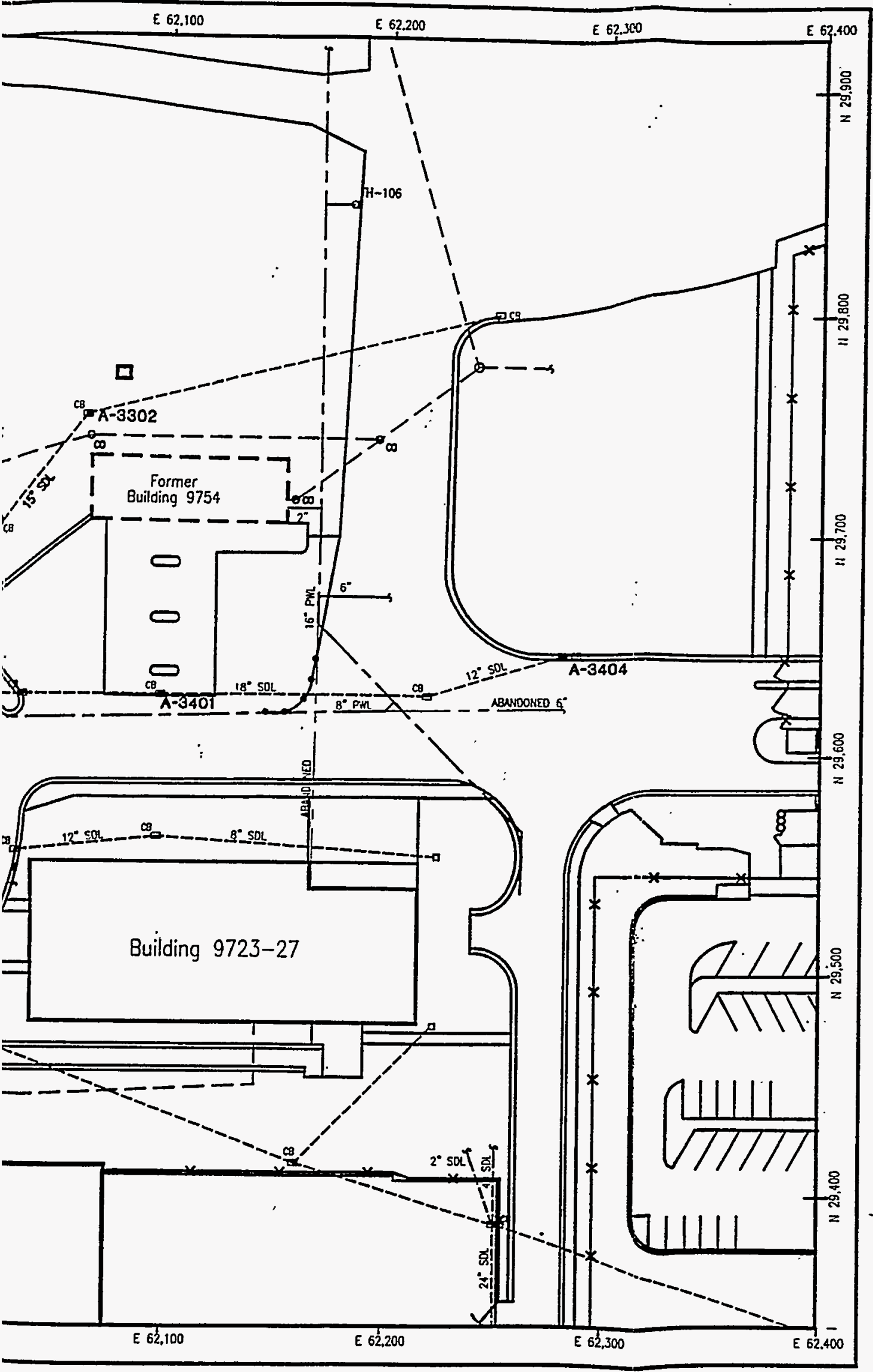




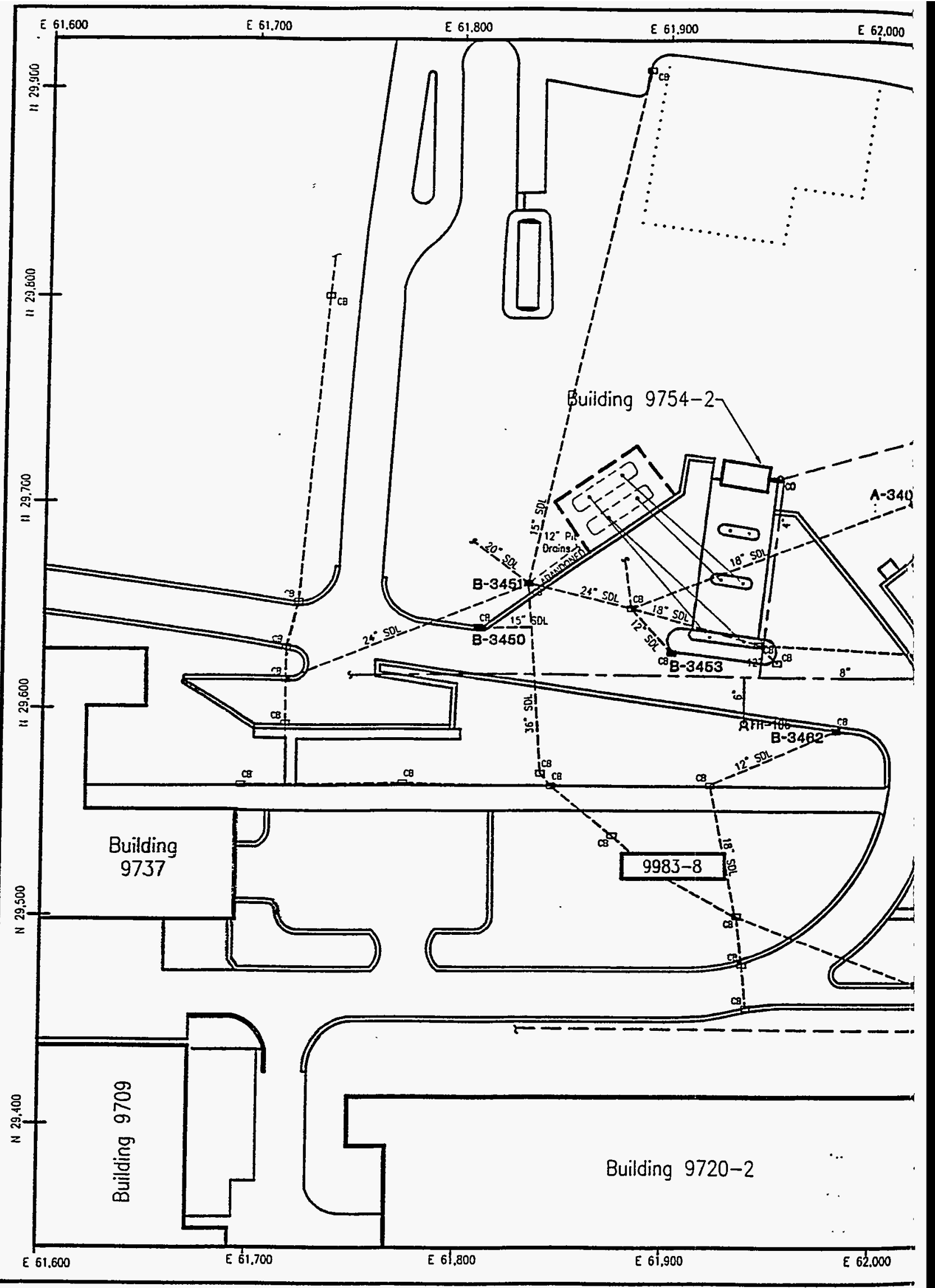

All location informotion presented in this figure is based upon LAES engineering drowings. results of previous LMES investigations, and/or field determinotions of

feature and sampling locotions. No representation or worranty, expressed or implied, is mode os to the occuracy of the information or stotements presented in this figure.

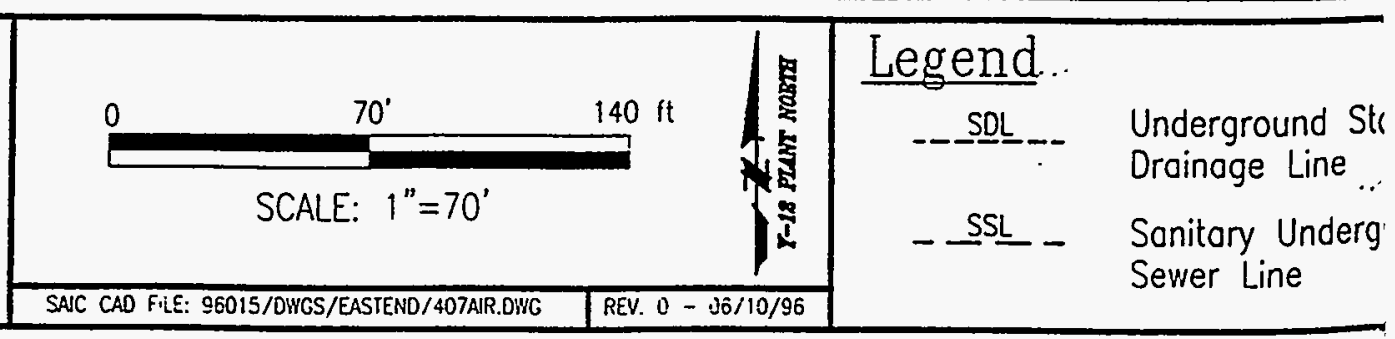


Table 3-1. Vapor Monitoring Results for Rust Garage Facility Site Status Monitoring, May 14, 1996

\begin{tabular}{ccccc}
\hline $\begin{array}{c}\text { Sampling } \\
\text { Location }\end{array}$ & $\begin{array}{c}\mathbf{O}_{2} \\
(\boldsymbol{\%})\end{array}$ & $\begin{array}{c}\text { LEL } \\
(\boldsymbol{\%})\end{array}$ & $\begin{array}{c}\text { Maximum } \\
\text { Organic Vapors } \\
\text { (ppm) }\end{array}$ & $\begin{array}{c}\text { Background } \\
\text { Organic Vapors } \\
\text { (ppm) }\end{array}$ \\
\hline A-3302 & 20.8 & 0 & 0.000 & 0.000 \\
A-3401 & 20.8 & 0 & 0.000 & 0.000 \\
A-3404 & 20.8 & 0 & 0.000 & 0.000 \\
B-3400 & 20.8 & 0 & 0.000 & 0.000 \\
B-3450 & 20.8 & 0 & 0.000 & 0.000 \\
B-3451 & 20.8 & 0 & 0.000 & 0.000 \\
B-3453 & 20.8 & 0 & 0.000 & 0.000 \\
B-3462 & 20.8 & 0 & 0.000 & 0.000 \\
F-305C & 20.8 & 0 & 0.000 & 0.000 \\
F-305C & 20.8 & 0 & 0.000 & 0.000 \\
F-3100 & 20.8 & 0 & 0.000 & 0.000 \\
F-3109 & 20.8 & 0 & 0.000 & 0.000 \\
\hline
\end{tabular}




\section{REFERENCES}

Energy Systems (Martin Marietta Energy Systems) 1992. Corrective Action Plan for Underground Storage Tanks 1219-U, 1222-U, 2082-U, and 2068-U at the Rust Garage Facility, Buildings 9720-15 and 9754-1, Appendix E, Martin Marietta Energy Systems, Inc. Y/SUB/9299928C/1.

Energy Systems 1994. Calendar Year 1993 Groundwater Quality Report for the Upper East Fork Poplar Creek Hydrologic Regime, Y-12 Plant, Oak Ridge, Tennessee: 1993 Groundwater Quality Data and Calculated Rate of Contaminant Migration.

Energy Systems 1994. Comprehensive Monitoring Report for Underground Storage Tanks $1219-\mathrm{U}, 1222-\mathrm{U}, 2082-\mathrm{U}$, and 2068-U at the Rust Garage Facility, Buildings $9720-15$ and 9754-1.

Harless, J., personal communication to R. Spence, March 7, 1994.

Jeffrey F., personal communication to R. Spence, May 6, 1996.

TDEC (Tennessee Department of Conservation) 1994. The TDEC UST Reference Handbook, 2nd ed. 
APPENDIX A

\section{LABORATORY ANALYTICAL RESULTS FOR} COMPREHENSIVE MONITORING (5/12/96) 


\section{ONCIASSIFIED}

OFFICIAL REPORT

IER Edward $M$ AMPLED: 05/12/96 09:23:00 ECEIVED: 05/13/96 $R: 013303$

ADDRESS Building 9115 Room 0128 M.S.8219 DATE NEEDED: 05/20/96 DATE COMPLETED : 05/24/96 SAMPLE DESCRIPTION: GRAB
CUSTOMER ID REQ NO LOCATION: GW-183 PROJECT CODE:
REQ NO SAMPLE NO E961330000
MTC STATUS

8601 APPROVED

CHARGE \#: S2205F03 CASE:

TS: FACILITY ID. 0-0101179754 AND 9754-2 (UST)

FINAL APPROVAL:

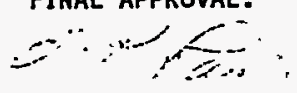

\begin{tabular}{|c|c|c|c|c|}
\hline $\begin{array}{l}\text { BTEX } \\
\text { TH: }\end{array}$ & $\begin{array}{l}\text { VOA Analysis, BTEX Compounds (SW846 8020) } \\
\text { PROC MTH: SW } 8468020 \text { PHASE: } \\
\text { THIS TEST WAS CANCELLED BY 4RH }\end{array}$ & & $\begin{array}{l}\text { REPLICATE: } 1 \\
\text { TIME ANALYZED: }\end{array}$ & $\begin{array}{l}\text { CANCELLED } \\
\text { APPROVER: }\end{array}$ \\
\hline $\begin{array}{l}\text { DRO_H } \\
\text { TH: }\end{array}$ & $\begin{array}{l}\text { TPH Diesel Range Organics Water (TN UST METHOD) } \\
\text { PROC MTH: TN UST METH PHASE: }\end{array}$ & & $\begin{array}{lr}\text { REPLICATE: } 1 & \text { STATUS: } \\
\text { TIME ANALYZED: } 05 / 21 / 96 \text { 13:15:00 }\end{array}$ & $\begin{array}{l}\text { APPROVED } \\
\text { APPROVER: E030124 }\end{array}$ \\
\hline MBER & DETERMINATION & DT & CONFIDENCE & UNIT \\
\hline 03 & $\begin{array}{l}\text { Diesel Range Organics } \\
\text { QUANTITATION LIMITS } \\
\text { DRO in Blank }\end{array}$ & & $\begin{array}{l}340 \\
100 \\
\text { NONE }\end{array}$ & $\begin{array}{l}\mathrm{ug} / \mathrm{L} \\
\mathrm{ug} / \mathrm{L} \\
\mathrm{ug} / \mathrm{L}\end{array}$ \\
\hline
\end{tabular}

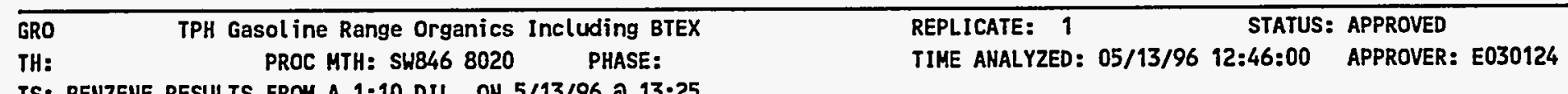

TS: BENZENE RESULTS FROM A 1:10 DIL. ON 5/13/96 a 13:25

\begin{tabular}{|c|c|c|c|c|c|}
\hline \multirow{2}{*}{ MBER } & DETERMINATION & DT & RESULT & CONFIDENCE & UNIT \\
\hline & Gasoline Range Organics & & 5300 & & $\mathrm{ug} / \mathrm{L}^{-}$ \\
\hline \multirow{7}{*}{7} & Benzene & & 1300 & & ug/L \\
\hline & Ethylbenzene & & 140 & & $u g / L$ \\
\hline & Toluene & & 990 & & ug/L \\
\hline & Xylene & & 390 & & ug/L \\
\hline & GRO Quantitation Limit & & 500 & & $u g / L$ \\
\hline & BTEX Quantitation Limit & & 50 & & ug/L \\
\hline & GRO Amount in Blank & & HONE & & ug/L \\
\hline
\end{tabular}


UNCIASSIFIED

OFFICIAL REPORT

76 13:50:24

Y-12 ANALYTICAL SERVICES ORGANIZATION

PAGE 1 OF 1

IER
$\begin{array}{lll}\text { Edward } M & \text { ADDRESS } & \\ \text { AMPLED: } 05 / 12 / 96 & 10: 39: 00 & \text { DATE MEEDED: 05/20/96 } \\ \text { SCEIVED: } 05 / 13 / 96 & \text { DATE COHPLETED: 05/24/96 } \\ \text { S: } 013303 & \text { SAMPLE DESCRIPTION: GRAB }\end{array}$

CUSTOMER ID REQ NO SAMPLE NO $\overline{E 961330002}$

LOCATION: GW-282

PROJECT CODE:
MTC $\quad$ STATUS
8601 APPROVED
CHARGE \#: S2205F03
CASE:

IS: FACILITY ID. 0-101179754 AND 9754-2 UST

\begin{tabular}{llll}
\hline BTEX & VOA Analysis, BTEX Compounds (SH846 8020) & REPLICATE: 1 STATUS: CANCELLED \\
TH: & PROC HTH: SH846 8020 PHASE: & TIME ANALYZED: &
\end{tabular}

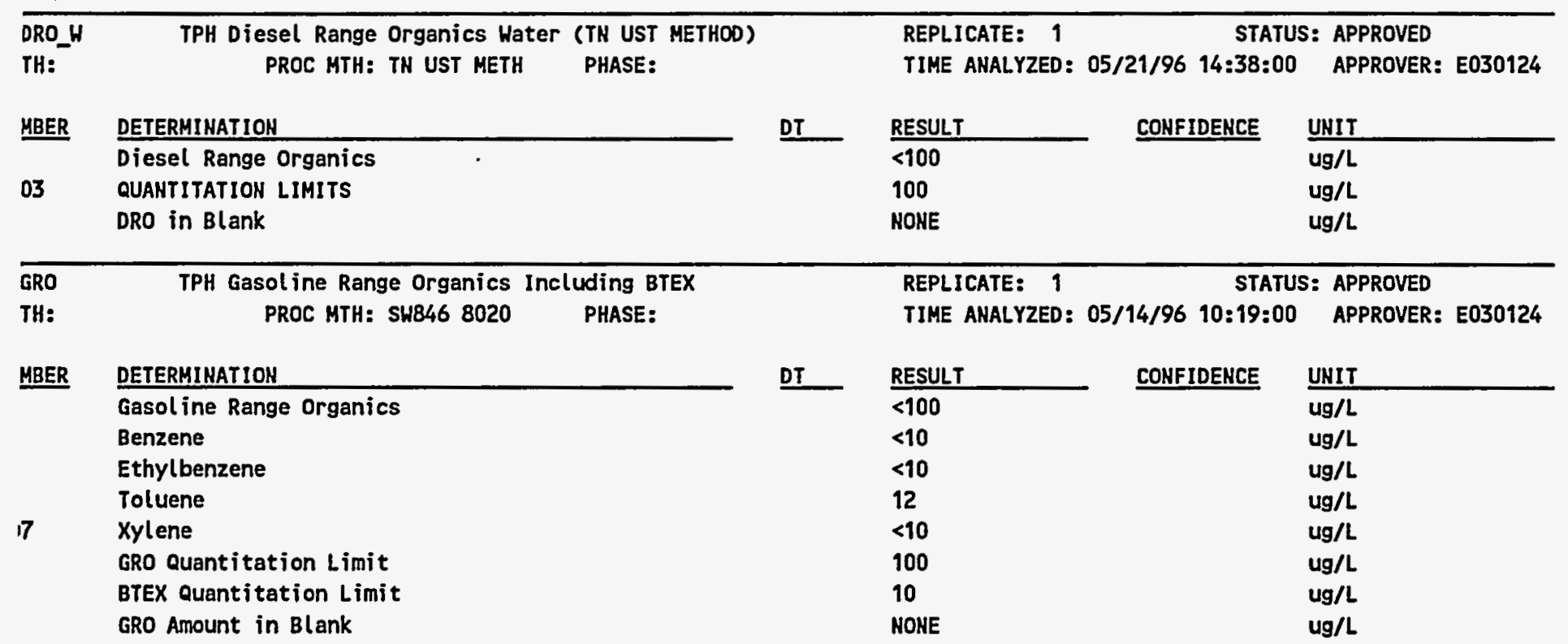




\section{UNCIASSIFIED}

OFFICIAL REPORT

\section{ITER}

$\pi$, Edward $M$ SAMPLED: 05/12/96 09:05:00 ZECEIVED: $05 / 13 / 96$

ER: 013303
CUSTOMER ID $\quad$ REQ NO $\frac{\text { SAMPLE NO }}{\text { E961330003 }}$

LOCATION: GH-283

PROJECT CODE: _. DATE COMPLETED: 05/24/96 SAMPLE DESCRIPTION: GRAB
MTC STATUS 8601 APPROVED CHARGE \#: S2205F03 CASE:

NTS: FACILITY ID. 0-010117

\begin{tabular}{llll}
\hline BTEX & VOA Analysis, BTEX Compounds (SH846 8020) & REPLICATE: 1 STATUS: CANCELLED \\
MTH: & PROC MTH: SH846 8020 PHASE: & TIME ANALYZED: & APPROVER:
\end{tabular}

THIS TEST WAS CANCELLED BY 4RW

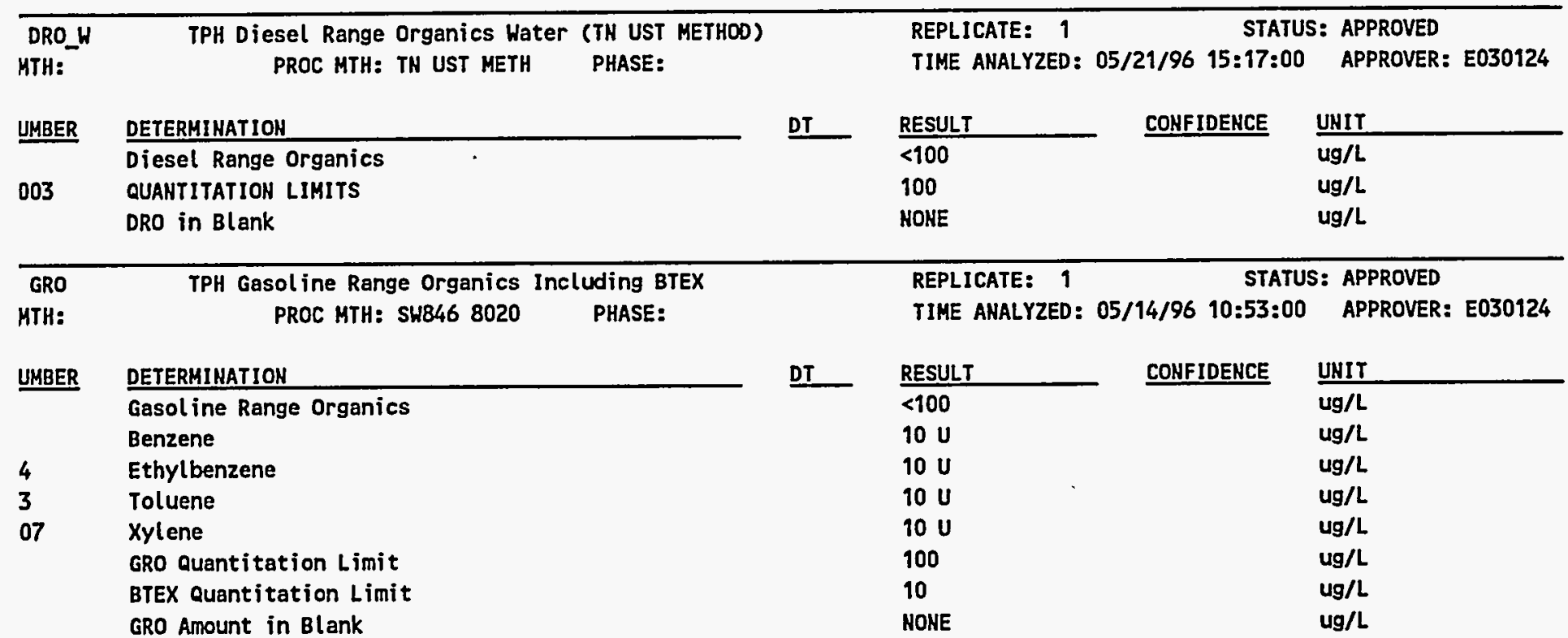




\section{UNCLASSIFIED}

OFFICIAL REPORT

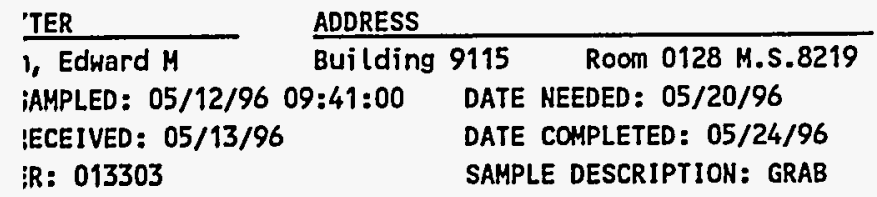

ITS: FACILITY ID. 0-0101179754 \& 9754-2 UST

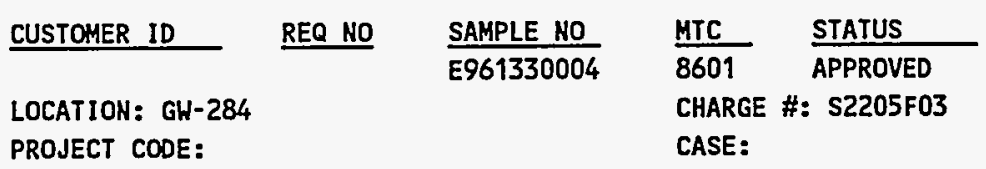

FINAL APPROVAL:
CASE:

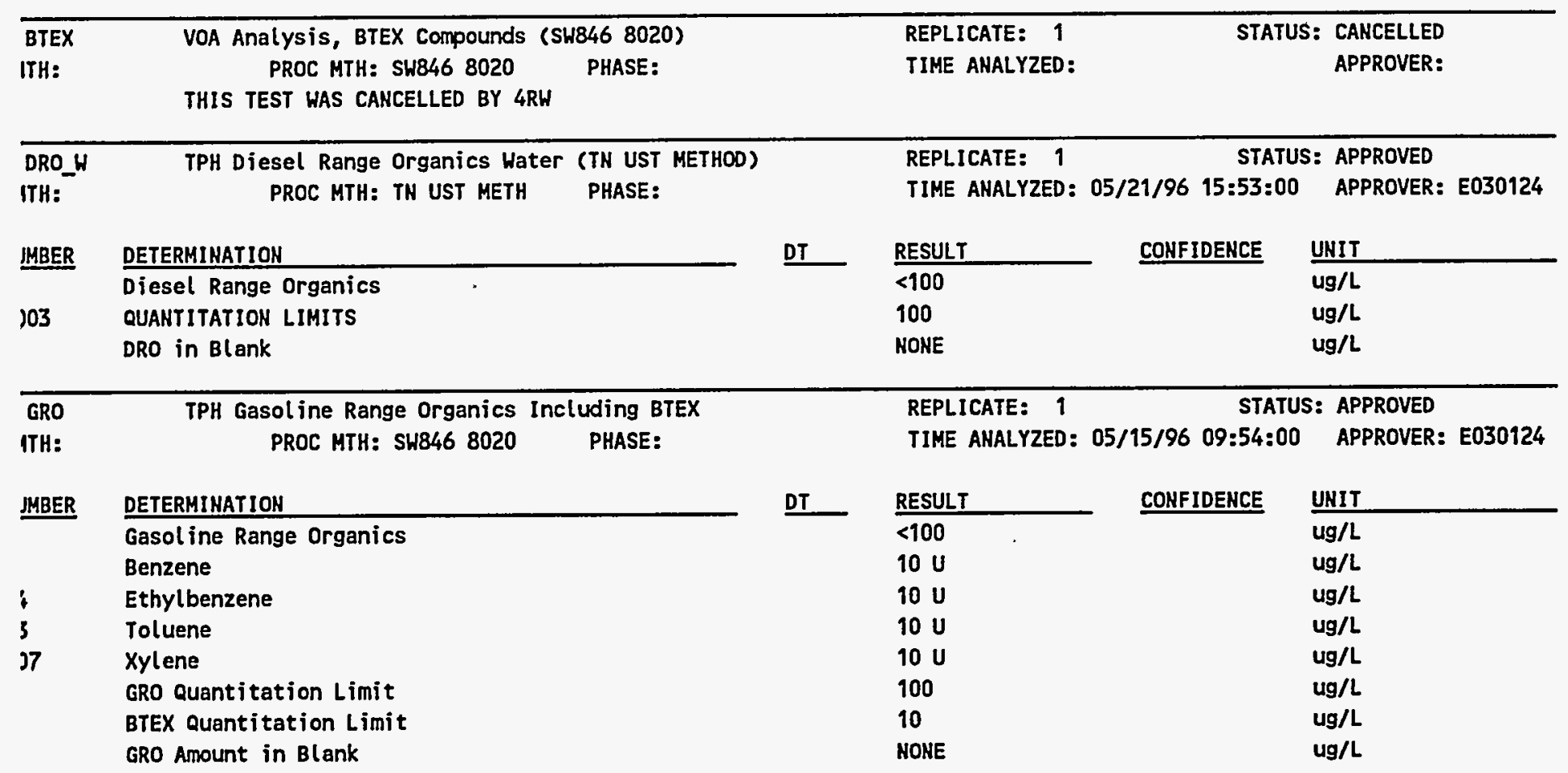


UNCLASSIFIED

OFFICIAL REPORT

'TER

1, Edward $M$ ADDRESS

Building 9115 Room 0128 M.S.8219 iAMPLED: 05/12/96 10:32:00 IECEIVED: 05/13/96 iR: 013303 DATE NEEDED: $05 / 20 / 96$ DATE COMPLETED: 05/24/96 SAMPLE DESCRIPTION: GRAB
CUSTOMER ID REQ NO SAMPLE NO

LOCATION: GW-285 PROJECT CODE:

\section{E961330005}

FINAL APPROVAL:
MTC STATUS $8601 \quad$ APPROVED CHARGE \#: S2205F03 CASE:

ITS: FACILITY ID. 0-0101179754 \& 9754-2 UST

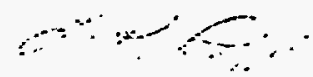

\begin{tabular}{llll}
\hline BTEX & VOA Analysis, BTEX Compounds (SW846 8020) & REPLICATE: 1 & STATUS: CANCELLED \\
ITH: & PROC MTH: SH846 8020 & PHASE: & TIME ANALYZED:
\end{tabular}

THIS TEST HAS CANCELLED BY 4RH

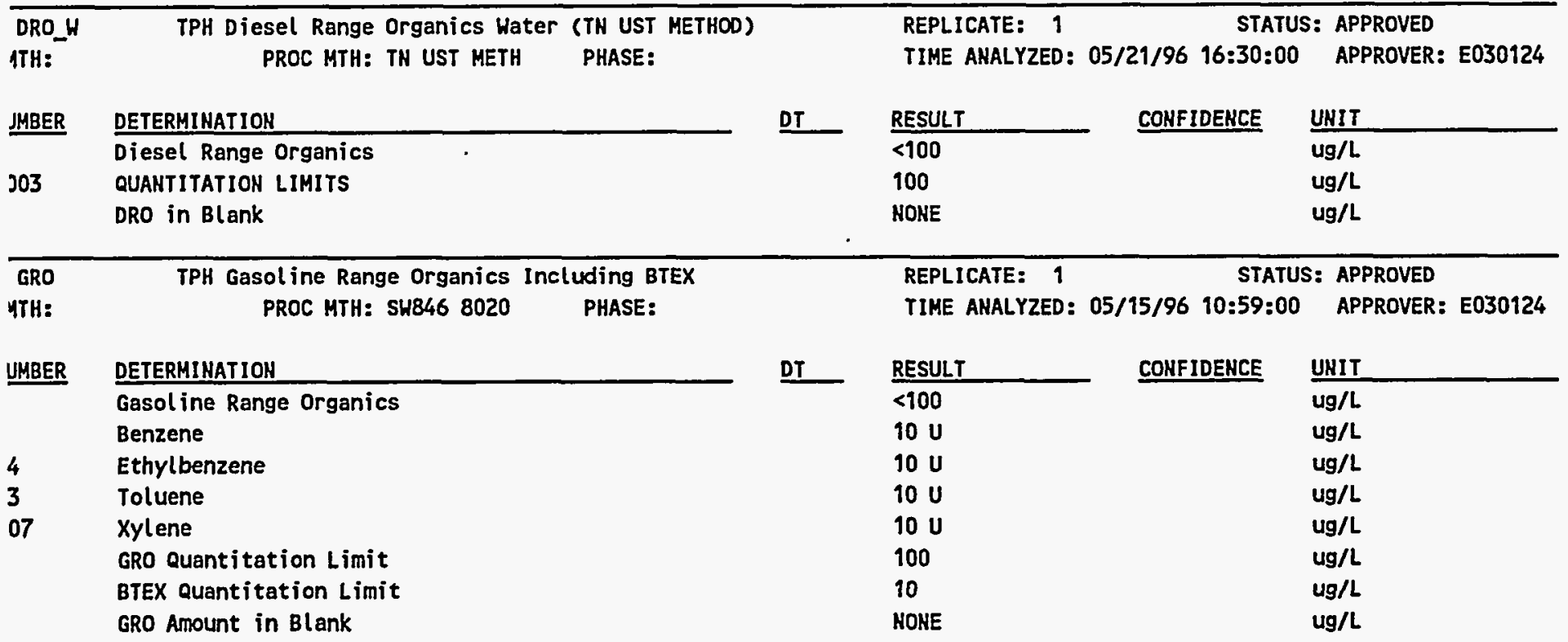




\section{UNCLASSIFIED}

OFFICIAL REPORT

$4 / 96 \quad 13: 52: 08$

ITTER

am, Edward $H$ SAMPLED: $05 / 12 / 96 \quad 14: 04: 00$ RECEIVED: $05 / 13 / 96$

ER: 013303
Y-12 ANALYTICAL SERVICES ORGANIZATION

CUSTOMER ID REQ NO SAMPLE NO E961330006

LOCATION: GW-658 PROJECT CODE:

PAGE 1 OF 1

ENTS: FACILITY ID. 0-0101179754\& 9754-2 UST

\begin{tabular}{llll}
\hline : BTEX & VOA Analysis, BTEX Compounds (SH846 8020) & REPLICATE: 1 STATUS: CANCELLED \\
MTH: & PROC HTH: SU846 8020 & PHASE: & TIME ANALYZED:
\end{tabular}

THIS TEST WAS CANCELLED BY 4RW

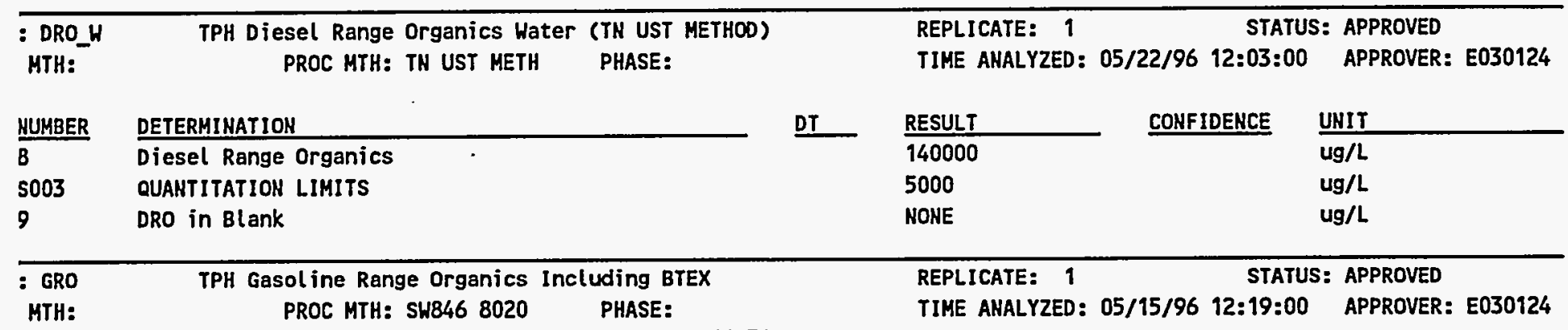

ENTS: ETHYLBENZENE RESULTS FROM A 1:10 DIL. ON 5/15/96 a 11:34

BENZENE RESULTS FROM A 1:100 DIL. ON 5/15/96 12:55

\begin{tabular}{|c|c|c|c|c|c|}
\hline MUMBER & DETERMINATION & DT & RESULT & CONFIDENCE & UNIT \\
\hline 4 & Gasol ine Range Organics & & 48000 & & $\mathrm{ug} / \mathrm{L}$ \\
\hline 2 & Benzene & & 13000 & & ug/L \\
\hline 14 & Ethylbenzene & & 1900 & & $\mathrm{ug} / \mathrm{L}$ \\
\hline 83 & Toluene & & 9400 & & $\mathrm{ug} / \mathrm{L}$ \\
\hline 207 & Xylene & & 11000 & & ug/L \\
\hline 2 & GRO Quantitation Limit & & 5000 & & ug/L \\
\hline 2 & BTEX Quantitation Limit & & 100 & & $\mathrm{ug} / \mathrm{L}$ \\
\hline .1 & GRO Amount in Blank & & NONE & . & $u g / L$ \\
\hline
\end{tabular}




\section{UNCLASSIFIED}

OFFICIAL REPORT

TER 1, Edward $M$

ADDRESS

Building 9115 Room 0128 M.S.8219

AMPLED: 05/12/96 14:20:00 DATE NEEDED: 05/20/96

ECEIVED: $05 / 13 / 96$

R: 013303
CUSTOMER ID REQ NO

LOCATION: GH-659

PROJECT CODE: $\frac{\text { SAMPLE NO }}{\text { E961330007 }}$

STATUS

8601 APPROVED

CHARGE \#: S2205F03

CASE:

ITS: FACILITY ID. 0-0101179754\& 9754-2 UST

\begin{tabular}{llll}
\hline BTEX & VOA Analysis, BTEX Compounds (SW846 8020) & REPLICATE: 1 STATUS: CANCELLED \\
ITH: & PROC MTH: SW846 $8020 \quad$ PHASE: & TIME AHALYZED: . & APPROVER:
\end{tabular}
THIS TEST WAS CANCELLED BY 4RH

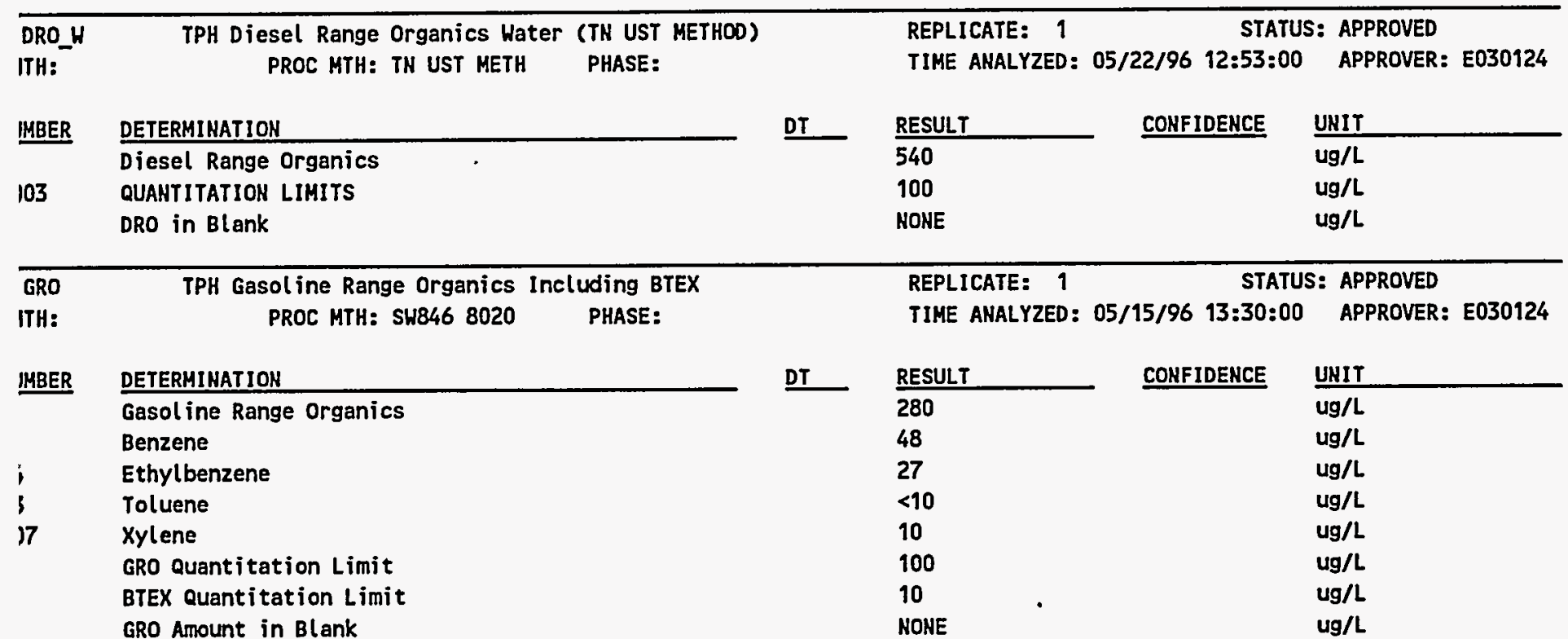

FIMAL-APPROVAL:-

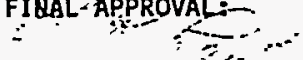




\section{UNCLASSIFIED}

OFFICIAL REPORT

\begin{tabular}{|c|c|c|}
\hline ITTER & ADDRESS & \\
\hline am, Edward $M$ & Building 9115 & Room 0128 H.S.8219 \\
\hline SAMPLED: $05 / 12 / 96$ & $5: 42: 00$ & JEEDED: $05 / 20 / 96$ \\
\hline RECEIVED: $05 / 13 / 96$ & DATE & COMPLETED: $05 / 24 / 96$ \\
\hline LER: 013303 & SAMP & DESCRIPTION: GRAB \\
\hline
\end{tabular}

CUSTOHER ID REQ NO SAMPLE NO E961330008

LOCATION: GH-767

PROJECT CODE: $\frac{\text { MTC }}{8601} \quad \frac{\text { STATUS }}{\text { APPROVED }}$ CHARGE \#: S2205F03 CASE:

ENTS: FACILITY ID. 0-0101179754 \& 9754-2 UST

FINAL APPROVAL:

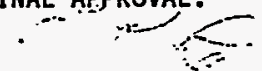

\begin{tabular}{llll}
\hline BTEX & VOA Analysis, BTEX Compounds (SW846 8020) & REPLICATE: 1 STATUS: CANCELLED \\
MTH: & PROC HTH: SH846 $8020 \quad$ PHASE: & TIME ANALYZED: & APPROVER:
\end{tabular}

THIS TEST HAS CANCELLED BY 4RH

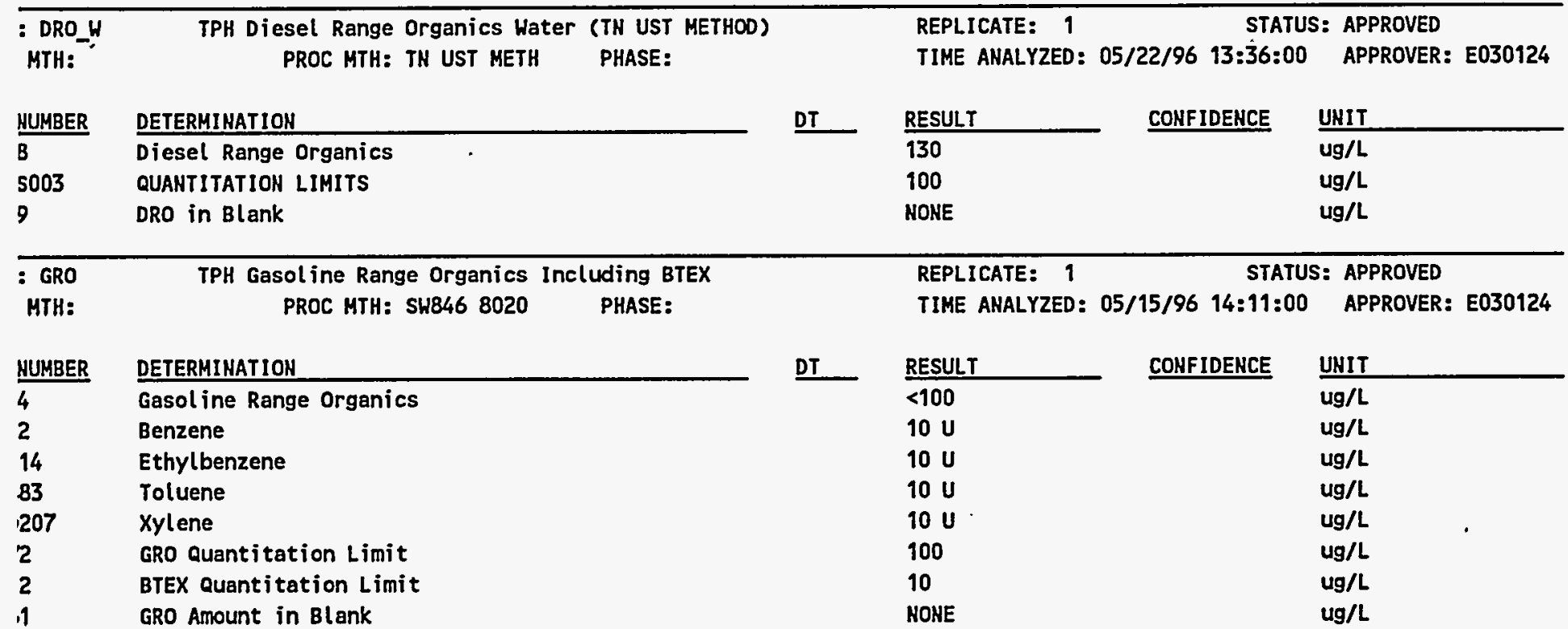




\section{UNCLASSIFIED}

OFFICIAL REPORT

\section{ITER}

$m$, Edward $M$ SAMPLED: 05/12/96 15:42:00 RECEIVED: $05 / 13 / 96$

ER: 013303

\section{ADDRESS}

Building 9115 Room 0128 M.S.8219

DATE NEEDED: 05/20/96

DATE COMPLETED: 05/24/96

SAMPLE DESCRIPTION: GRAB
CUSTOMER ID REQ NO $\frac{\text { SAMPLE NO }}{\text { E961330009 }}$

LOCATION: GH-802

PROJECT CODE:
MTC STATUS 8601 APPROVED CHARGE \#: S2205F03 CASE:

NTS: FACILITY ID. 0-0101179754\& 9754-2 UST

FINAL APPROVAL:

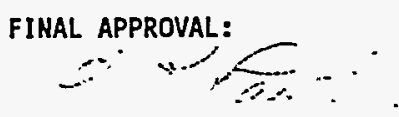

\begin{tabular}{llll}
\hline BTEX & VOA Analysis, BTEX Compounds (SW846 8020) & REPLICATE: 9 STATUS: CANCELLED \\
HTH: & PROC HTH: SH846 8020 & PHASE: & TIME ANALYZED:
\end{tabular}

THIS TEST HAS CANCELLED BY 4 RW

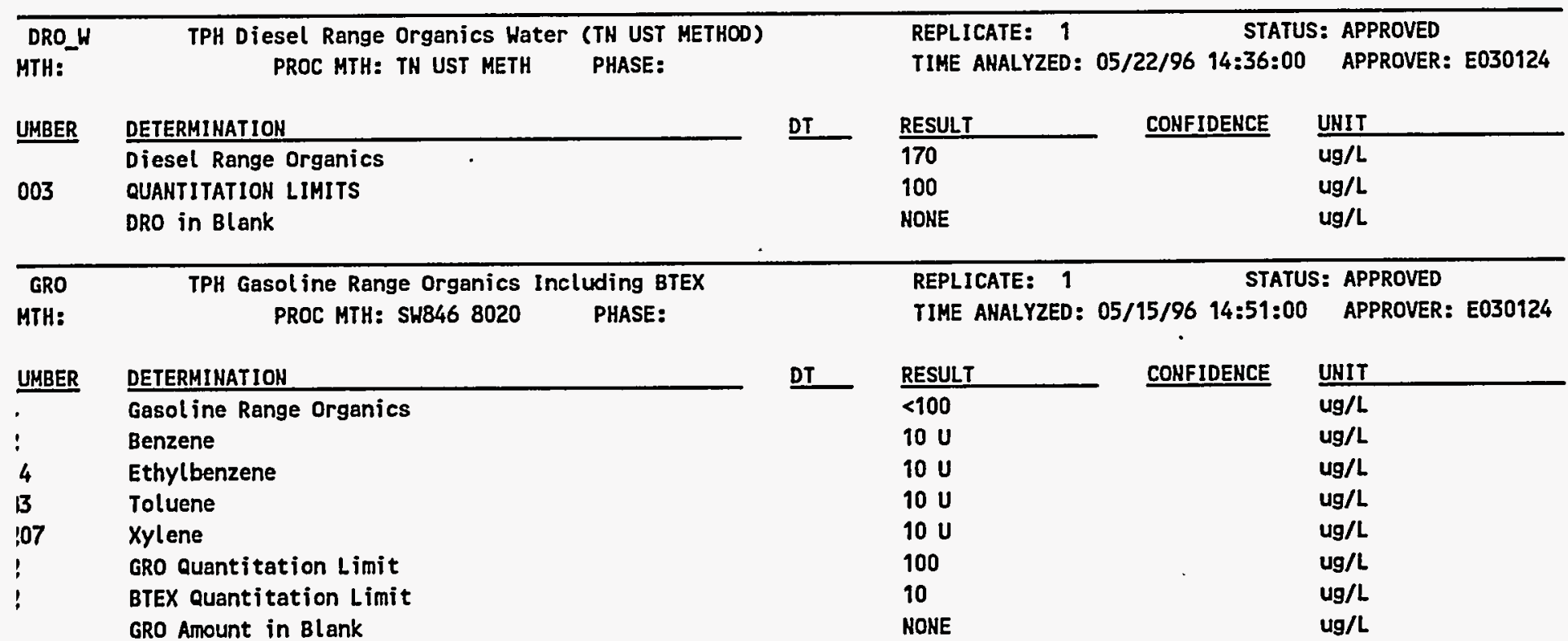




\section{UNCLASSIFIED}

OFFICIAL REPORT

\section{TTER} in, Edward $M$ SAMPLED: 05/12/96 16:20:00 RECEIVED: 05/13/96 ER: 013303

\section{ADDRESS} Building 9115 Room 0128 M.S.8219 DATE NEEDED: 05/20/96 DATE COMPLETED: 05/24/96 SAMPLE DESCRIPTION: GRAB
CUSTOMER ID REQ NO SAMPLE NO E961330010

LOCATION: GW-803

PROJECT CODE:
MTC STATUS

8601 APPROVED

CHARGE \#: S2205F03 CASE:

:NTS: FACILITY ID. 0-0101179754 \& 9754-2 UST

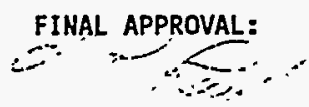

\begin{tabular}{llll}
\hline BTEX & VOA Analysis, BTEX Compounds (SW846 8020) & REPLICATE: 1 STATUS: CANCELLED \\
MTH: & PROC HTH: SH846 $8020 \quad$ PHASE: & TIME ANALYZED: &
\end{tabular}

THIS TEST HAS CANCELLED BY $4 R W$

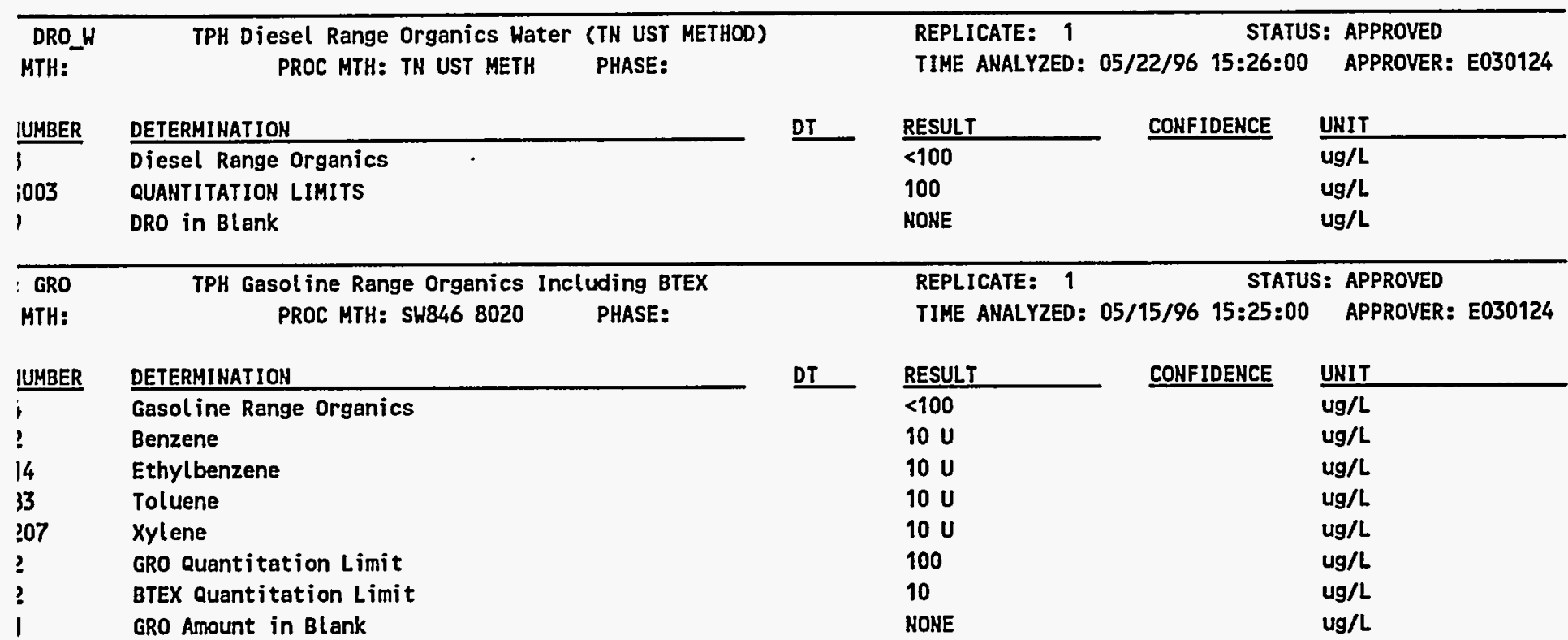


OFFICIAL REPORT

$1 / 96 \quad 13: 53: 03$

TTER

im, Edward $M$ RECEIVED: $05 / 13 / 96$

ER: 013303

:NTS: FACILITY ID. 0-010117 $9754 \& 9754-2$ UST
Y-12 ANALYTICAL SERVICES ORGANIZATION E961330011

LOCATION: GW-804 PROJECT CODE:

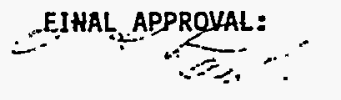

PAGE 1 OF 1

MTC STATUS

8601 APPROVED

CHARGE \#: S2205F03

CASE:

\begin{tabular}{llll}
\hline BTEX & VOA Analysis, BTEX Compounds (SW846 8020) & REPLICATE: 1 STATUS: CANCELLED \\
MTH: & PROC MTH: SH846 $8020 \quad$ PHASE: & TIME ANALYZED: & APPROVER:
\end{tabular}

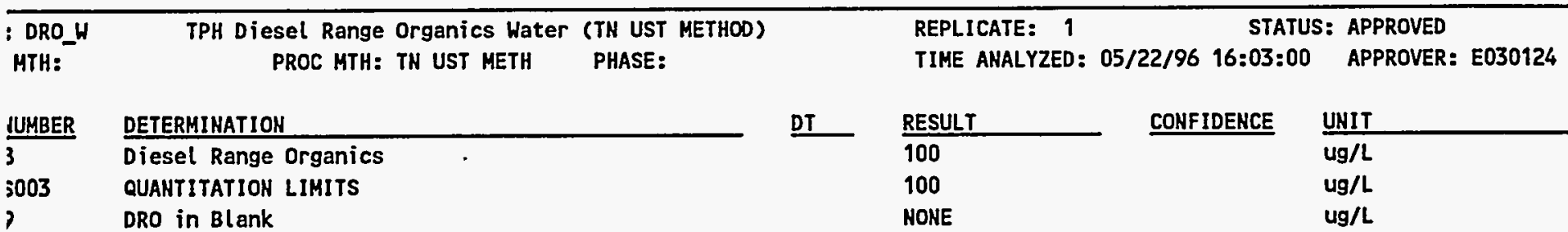

?

DRO in Blank

REPLICATE: 1 STATUS: APPROVED

: GRO TPH Gasol ine Range Organics Including BTEX

TIME ANALYZED: 05/16/96 11:17:00 APPROVER: E030124

MTH: PROC MTH: SH846 8020 PHASE:

DETERHINATIOH

DT

\begin{tabular}{l} 
RESULT \\
\hline$<100$ \\
$10 \mathrm{U}$ \\
$10 \mathrm{U}$ \\
$10 \mathrm{U}$ \\
$10 \mathrm{U}$ \\
100 \\
10 \\
NOME
\end{tabular}

CONFIDENCE UHIT

Gasoline Range Organics

Benzene

Ethylbenzene

Toluene

Xylene

GRO Quantitation Limit

BTEX Quantitation Limit

GRO Amount in Blank

HONE

ug/L

ug/L

ug/L

ug/L

$u g / L$

ug/L

ug/L

ug/L 
UNCLASSIFIED

OFFICIAL REPORT

TER

\section{, Edward $\mathrm{H}$} ECEIVED: $05 / 13 / 96$ R: 013303

\section{ADDRESS}

Building 9115 Room 0128 H.S.8219

DATE NEEDED: 05/20/96

DATE COMPLETED: 05/24/96

SAMPLE DESCRIPTION: GRAB
CUSTOMER ID $\quad$ REQ NO $\frac{\text { SAMPLE NO }}{\text { E961330012 }}$

LOCATION: GW-183 PROJECT COOE:
MTC STATUS 8601 APPROVED CHARGE \#: S2205F03 CASE:

TS: FACILITY ID. 0-0101179754\& 9754-2 UST

FINAL APPROVAL:

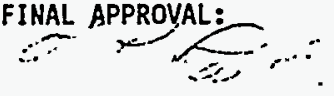

\begin{tabular}{llll}
\hline BTEX & VOA Analysis, BTEX Compounds (SH846 8020) & REPLICATE: 1 & STATUS: CANCELLED \\
TH: & PROC MTH: SW846 8020 & PHASE: & TIME ANALYZED:
\end{tabular}

THIS TEST WAS CANCELLED BY $4 R \mathrm{RH}$

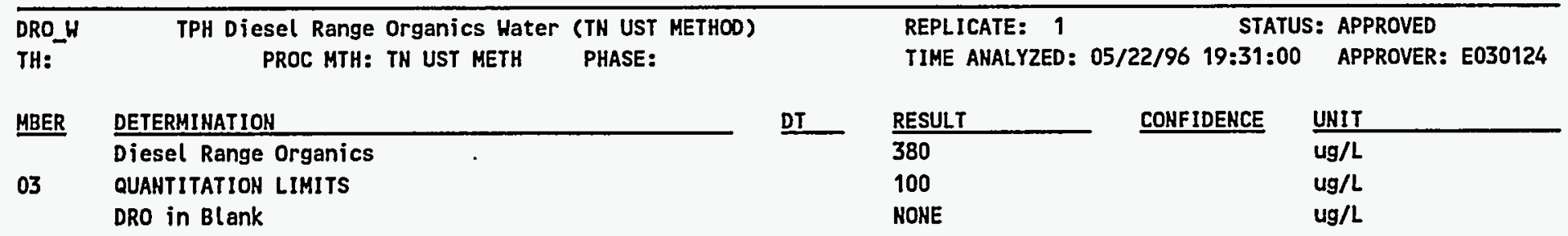

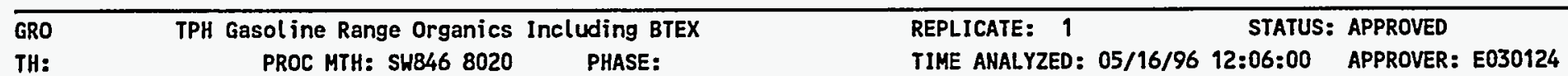

TS: BENZENE RESULTS ARE FROM A 1:10 DIL. ON 5/16/96 a 12:40

\begin{tabular}{|c|c|c|c|c|c|}
\hline MBER & DETERMINATION & DT & RESULT & CONFIDENCE & UNIT \\
\hline & Gasoline Range Organics & & 4900 & & $\mathrm{ug} / \mathrm{L}$ \\
\hline & Benzene & & 1300 & & ug/L \\
\hline , & Ethylbenzene & & 140 & & ug/L \\
\hline & Toluene & & 950 & & $u g / L$ \\
\hline 17 & Xylene & & 360 & & ug/L \\
\hline & GRO Quantitation Limit & & 500 & & ug/L \\
\hline & BTEX Quantitation Limit & & 50 & & ug/L \\
\hline & GRO Amount in Blank & & HONE & & $u g / L$ \\
\hline
\end{tabular}




\section{UNCLASSIFIED}

OFFICIAL REPORT

TTER

m, Edward $H$ SAMPLED: 05/12/96 $16: 20$ RECEIVED: $05 / 13 / 96$ ER: 013303

$$
\text { ADDRESS }
$$

Building 9115 Room 0128 M.S.8219 DATE NEEDED: 05/20/96 DATE COMPLETED: 05/24/96 SAMPLE DESCRIPTION: GRAB
CUSTOMER ID REQ NO SAMPLE NO E961330013

LOCATION: GH-803 PROJECT CODE:
MTC STATUS 8601 APPROVED CHARGE \#: S2205F03 CASE:

NTS: FACILITY ID. 0-0101179754\& 9754-2 UST

FINAL APPROVAL:

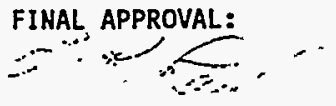

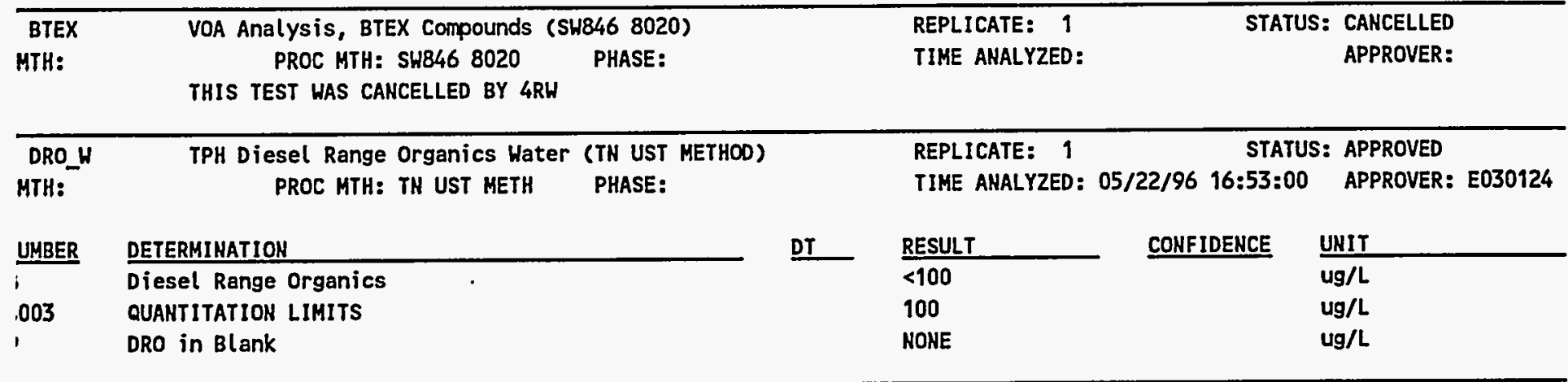

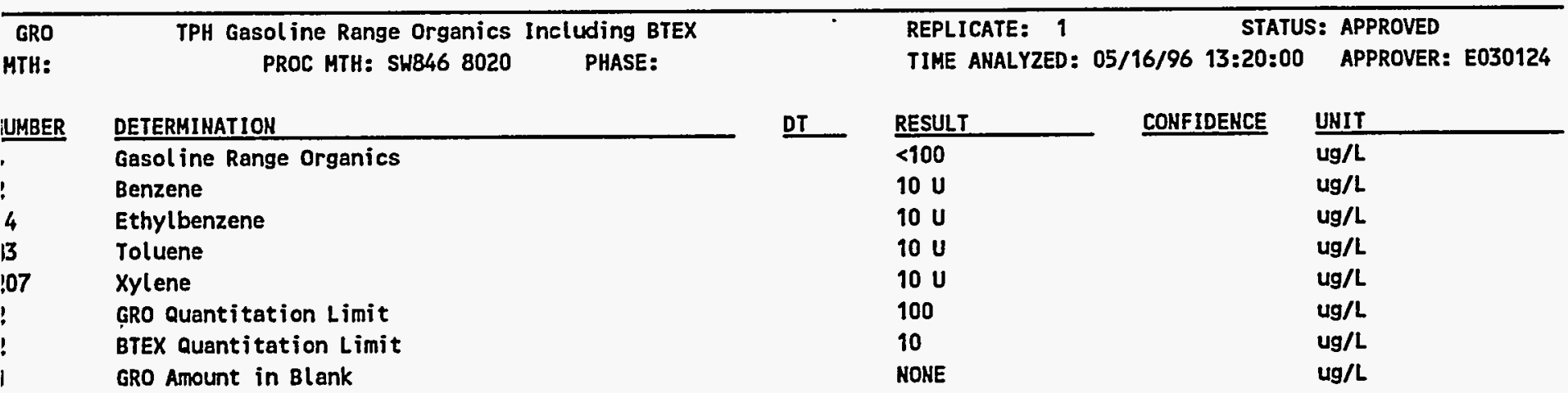




\section{UNCLASSIFIED}

OFFICIAL REPORT

\section{TTER}

$m$, Edward $M$

ADDRESS

Building 9115 Room 0128 M.S.8219

SAMPLED: 05/12/96 08:30:00 DATE NEEDED: 05/20/96

RECEIVED: $05 / 13 / 96$

ER: 013303
CUSTOMER ID REQ NO

LOCATION: GW-183-B

PROJECT CODE:
SAMPLE NO MTC STATUS E961330014 $8601 \quad$ APPROVED

CHARGE \#: S2205F03

CASE:

HTS: FACILILTY ID. 0-0101179754\& 9754-2 UST

FINAL APPROVAL:

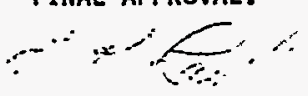

\begin{tabular}{llll}
\hline BTEX & VOA Analysis, BTEX Compounds (SW846 8020) & REPLICATE: '1 & STATUS: CANCELLED \\
MTH: & PROC MTH: SW846 8020 PHASE: & TIHE ANALYZED: & APPROVER: \\
& THIS TEST WAS CANCELLED BY 4RW &
\end{tabular}

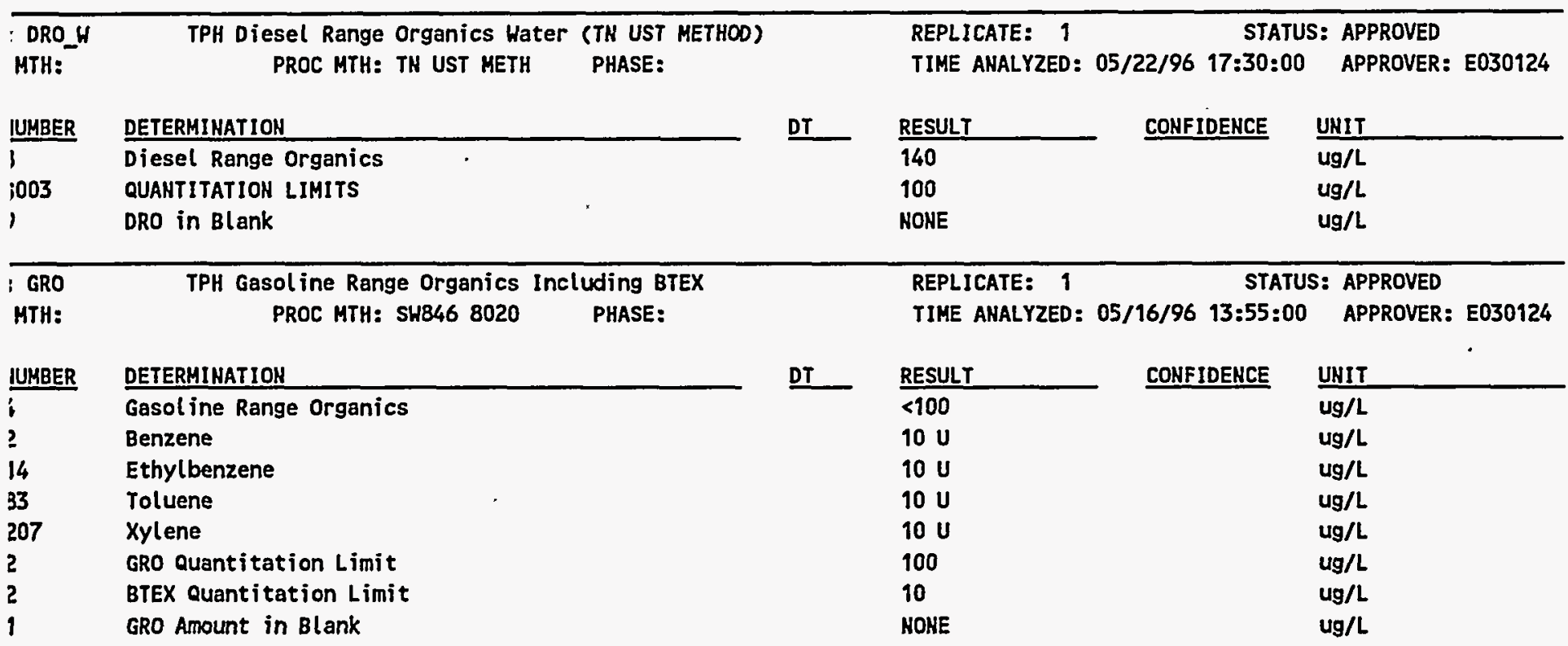




\section{UNCLASSIFIED}

OFFICIAL REPORT

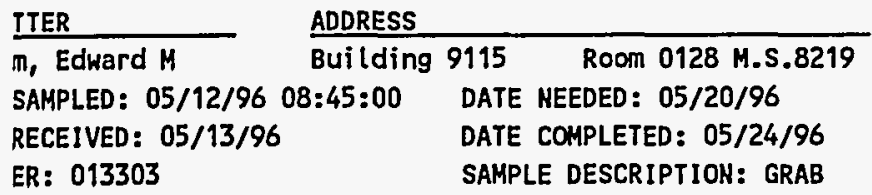

ROOM 0128 M.S.
DATE COMPLETED: 05/24/96

SAMPLE DESCRIPTION: GRAB

NTS: FACILITY ID. 0-0101179754\& 9754-2 UST

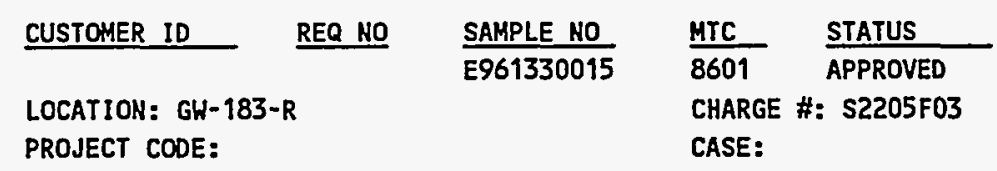

FIMAL APPROVAL:

\begin{tabular}{|c|c|c|c|c|}
\hline $\begin{array}{l}\text { BTEX } \\
\text { MTH: }\end{array}$ & $\begin{array}{l}\text { VOA Analysis, BTEX Compounds (SW846 8020) } \\
\text { PROC MTH: SH846 } 8020 \text { PHASE: } \\
\text { THIS TEST WAS CANCELLED BY 4RW }\end{array}$ & & $\begin{array}{l}\text { REPLICATE: } 1 \\
\text { TIME ANALYZED: }\end{array}$ & $\begin{array}{l}\text { CANCELLED } \\
\text { APPROVER: }\end{array}$ \\
\hline $\begin{array}{l}\text { DRO_H } \\
\text { MTH: }\end{array}$ & $\begin{array}{c}\text { TPH Diesel Range Organics Water (TN UST METHOD) } \\
\text { PROC MTH: TN UST METH PHASE: }\end{array}$ & & $\begin{array}{l}\text { REPLICATE: } 1 \\
\text { TIME ANALYZED: } 05 / 22 / 96 \text { STATUS: } \\
18: 06: 00\end{array}$ & $\begin{array}{l}\text { S: APPROVED } \\
\text { APPROVER: E030124 }\end{array}$ \\
\hline UMBER & $\begin{array}{l}\text { DETERMINATION } \\
\text { Diesel Range Organics } \\
\text { QUANTITATION LIMITS } \\
\text { DRO in Blank }\end{array}$ & DT & $\begin{array}{ll}\text { RESULT } & \text { CONFIDENCE } \\
<100 & \\
100 & \end{array}$ & $\begin{array}{l}\text { UNIT } \\
\text { ug/L } \\
\text { ug/L } \\
\text { ug/L }\end{array}$ \\
\hline $\begin{array}{l}\text { GRO } \\
\text { MTH: }\end{array}$ & $\begin{array}{c}\text { TPH Gasoline Range organics including BTEX } \\
\text { PROC MTH: SHB46 } 8020 \quad \text { PHASE: }\end{array}$ & & $\begin{array}{lr}\text { REPLICATE: } 1 & \text { STATUS: } \\
\text { TIME ANALYZED: } & 05 / 16 / 96 \quad 14: 30: 00\end{array}$ & $\begin{array}{l}\text { S: APPROVED } \\
\text { APPROVER: E030124 }\end{array}$ \\
\hline UMBER & DETERMINATION & DT & CONFIDENCE & UNIT \\
\hline $\begin{array}{l}4 \\
3 \\
: 07 \\
\vdots\end{array}$ & $\begin{array}{l}\text { Gasol ine Range Organics } \\
\text { Benzene } \\
\text { Ethylbenzene } \\
\text { Toluene } \\
\text { Xylene } \\
\text { GRO Quantitation Limit } \\
\text { BTEX Quantitation Limit } \\
\text { GRO Amount in Blank }\end{array}$ & & $\begin{array}{l}<100 \\
10 \mathrm{U} \\
10 \mathrm{U} \\
10 \mathrm{U} \\
10 \mathrm{U} \\
100 \\
10 \\
\text { NONE }\end{array}$ & $\begin{array}{l}u g / L \\
u g / L \\
u g / L \\
u g / L \\
u g / L \\
u g / L \\
u g / L \\
u g / L\end{array}$ \\
\hline
\end{tabular}




\section{ONCLASSIEIED}

OFFICIAL REPORT

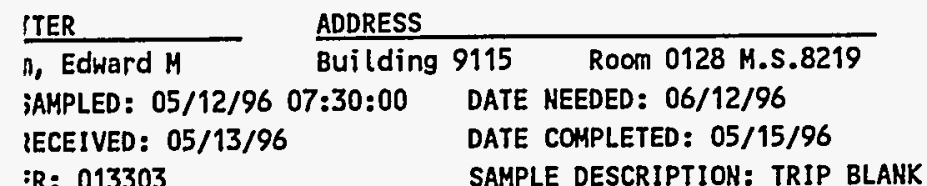

:R: 013303

ITS: FACILITY ID. 0-0101179754\& 9754-2 UST

\section{CUSTOMER ID $\quad$ REQ NO $\quad \frac{\text { SAMPLE NO }}{\text { E961330016 }}$ \\ LOCATION: 9207 PROJECT CODE:

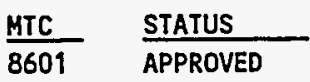 \\ CHARGE \#: S2205F03 \\ CASE:}

FINAL APPROVAL:

\begin{tabular}{|c|c|c|c|c|c|c|}
\hline \multicolumn{3}{|c|}{ Volatile Organics by GC/MS (EPA 624) } & \multirow[b]{3}{*}{ DT } & \multirow{2}{*}{$\begin{array}{l}\text { REPLICATE: } 1 \\
\text { TIME ANALYZED: }\end{array}$} & \multicolumn{2}{|c|}{ STATUS: APPROVED } \\
\hline 1TH: & PROC MTH: EPA 624 & PHASE: & & & 05/13/96 10:54:00 & APPROVER: E030124 \\
\hline \multirow[t]{9}{*}{ JMBER } & DETERMINATION & & & RESULT & CONFIDENCE & UNIT \\
\hline & Chloromethane & & & $10 \mathrm{U}$ & & ug/L \\
\hline & Bromomethane & & & $10 u$ & & $u g / L$ \\
\hline & Vinyl chloride & & & $10 \mathrm{U}$ & & $u g / L$ \\
\hline & Chloroethane & & & $10 \mathrm{U}$ & & ug/L. \\
\hline & Trichlorofluoromethane & & & $10 \mathrm{U}$ & & $u g / L$ \\
\hline & Methylene chloride & & & $10 \mathrm{U}$ & & $u g / L$ \\
\hline & 1,1-Dichloroethene & & & $10 \mathrm{U}$ & & ug/L \\
\hline & 1,1-Dichloroethane & & & $10 \mathrm{U}$ & & ug/L \\
\hline \multirow[t]{2}{*}{; } & trans-1,2-Dichloroethene & & & $10 \mathrm{U}$ & & ug/L \\
\hline & Chloroform & & & $10 \mathrm{U}$ & & ug/L \\
\hline \multirow[t]{4}{*}{$?$} & 1,2-Dichloroethane & & & $10 \mathrm{U}$ & & $\mathbf{u g} / \mathrm{L}$ \\
\hline & $1,1,1$-Trichloroethane & & & $10 U$ & - & ug/L \\
\hline & Carbon tetrachloride & & & $10 \mathrm{U}$ & & ug/L \\
\hline & Bromodichloromethane & & & $10 \mathrm{U}$ & & ug/L \\
\hline \multirow[t]{2}{*}{ B } & 2-Chloroethylvinyl ether & & & $10 U$ & & ug/L \\
\hline & 1,2-Dichloropropane & & & $10 \mathrm{U}$ & & ug/L \\
\hline \multirow[t]{2}{*}{015} & cis-1,3-Dichloropropene & & & $10 \mathrm{U}$ & & ug/L \\
\hline & Trichloroethene & & & $10 \mathrm{U}$ & & ug/L \\
\hline \multirow[t]{3}{*}{1} & Dibromochloromethane & & & $10 U$ & & $\mathrm{ug} / \mathrm{L}$ \\
\hline & 1,1,2-Trichloroethane & & & $10 \mathrm{U}$ & & ug/L \\
\hline & Benzene & & & $10 \mathrm{U}$ & & ug/L \\
\hline \multirow[t]{2}{*}{026} & trans-1,3-Dichloropropene & & & $10 \mathrm{U}$ & & ug/L \\
\hline & Bromoform & & & $10 \mathrm{U}$ & & $\mathrm{ug} / \mathrm{L}$ \\
\hline \multirow[t]{2}{*}{4} & Tetrachloroethene & & & $10 \mathrm{U}$ & & $u g / L$ \\
\hline & $1,1,2,2,-$ Tetrachloroethane & & & $10 \mathrm{U}$ & & $u g / L$ \\
\hline 3 & Toluene & & & $10 \mathrm{U}$ & & $u g / L$ \\
\hline 7 & Chlorobenzene & & & $10 \mathrm{U}$ & & $u g / L$ \\
\hline 4 & Ethylbenzene & & & $10 \mathrm{U}$ & & ug/L \\
\hline
\end{tabular}




\section{DISTRIBUTION}

Health, Safety, Environment, and Accountability Organization

U.S. Department of Energy

E.M. Ingram (2)

S.L. Lee

R.L. Johnson, Jr.

R.J. Spence/L.M. Sparks

File - EMD

W.B. Mansel

HSEA Document Center

Facility Management Organization

A.K. Lee/DOE-OSTI (2)

Y-12 Central Files - RC

A.E. Hodge

Environmental Compliance

Organization

C.L. Stair

Tennessee Department of Environment and Conservation

C. Head

E.C. Leming/J.D. Harless 